TRANSACTIONS OF THE

AMERICAN MATHEMATICAL SOCIETY

Volume 353, Number 2, Pages 809-838

S 0002-9947(00)02702-1

Article electronically published on October 19, 2000

\title{
BOUNDARY VALUE PROBLEMS FOR HIGHER ORDER PARABOLIC EQUATIONS
}

\author{
RUSSELL M. BROWN AND WEI HU
}

This paper is dedicated to Gene Fabes

\begin{abstract}
We consider a constant coefficient parabolic equation of order $2 m$ and establish the existence of solutions to the initial-Dirichlet problem in cylindrical domains. The lateral data is taken from spaces of Whitney arrays which essentially require that the normal derivatives up to order $m-1$ lie in $L^{2}$ with respect to surface measure. In addition, a regularity result for the solution is obtained if the data has one more derivative. The boundary of the space domain is given by the graph of a Lipschitz function. This provides an extension of the methods of Pipher and Verchota on elliptic equations to parabolic equations.
\end{abstract}

\section{INTRODUCTION}

Consider a parabolic operator $D_{t}+L$ on $\mathbf{R}^{n} \times(0, \infty), n \geq 2$, where

$$
L=\sum_{|a|=|\beta|=m}(-1)^{m} a_{\alpha \beta} D^{\alpha} D^{\beta}
$$

is an elliptic operator of order $2 m, m \geq 2$, on $\mathbf{R}^{n}$. We assume that $L$ has real, constant coefficients and, without loss of generality, we may assume that the coefficients satisfy the symmetry condition

$$
a_{\alpha \beta}=a_{\beta \alpha} .
$$

The operator is elliptic, which means that there is a constant $E>0$ so that

$$
a_{\alpha \beta} \xi^{\alpha} \xi^{\beta} \geq E|\xi|^{2 m}, \quad \xi \in \mathbf{R}^{n} .
$$

To simplify the notation, we assume that the indices $\alpha$ and $\beta$ appearing in the operator are summed.

Our main interest is the initial-Dirichlet problem

$$
\begin{cases}\left(D_{t}+L\right) u(X, t)=0, & X \in \Omega, t>0, \\ D^{\alpha} u(Q, t)=\psi_{\alpha}(Q, t), & |\alpha| \leq m-1, Q \in \partial \Omega, t>0, \\ u(X, 0)=0, & X \in \Omega .\end{cases}
$$

The domain $\Omega$ is the region above the graph of a function, $\Omega=\left\{X_{n}>\phi\left(X^{\prime}\right)\right\}$ with $\phi: \mathbf{R}^{n-1} \rightarrow \mathbf{R}$ a Lipschitz function satisfying $\|\nabla \phi\|_{L^{\infty}} \leq M$. Here and below, we write $X \in \mathbf{R}^{n}$ as $\left(X^{\prime}, X_{n}\right) \in \mathbf{R}^{n-1} \times \mathbf{R}$ when we need to distinguish the last

Received by the editors June 2, 1998.

2000 Mathematics Subject Classification. Primary 35K35.

(C)2000 American Mathematical Society 
variable. We let $S_{T}=\partial \Omega \times(0, T)$ denote the lateral boundary of the cylinder $\Omega \times(0, T)$. We let $\Lambda$ be a fractional time derivative given by

$$
\widehat{\Lambda f}(Q, \tau)=(i \tau)^{1 / 2 m} \hat{f}(Q, \tau),
$$

where a caret indicates the partial Fourier transform in the time variable (see section 6 for a more detailed definition of the operator $\Lambda$ ).

Now we define spaces on $S_{\infty}$ from which we will take boundary data. Note that the functions $\psi_{\alpha}$ in (1.3) cannot be specified arbitrarily. In fact, the array of functions must satisfy compatibility conditions which guarantee that they arise as derivatives of a single function. It seems simplest to use this as the definition of our spaces of boundary data. Thus, if $\psi \in C_{0}^{\infty}\left(\mathbf{R}^{n} \times(0, \infty)\right)$ we let $\vec{\psi}=\left(\psi_{\alpha}\right.$ : $|\alpha| \leq k$ ) denote the array of partial derivatives in the spatial variables, $X$, where $\psi_{\alpha}=\left.D^{\alpha} \psi\right|_{S_{\infty}}$. We call $\vec{\psi}$ the Whitney array of order $k$ generated by $\psi$. We define a norm on this space by

$$
\|\vec{\psi}\|_{W A_{k}^{2}\left(S_{\infty}\right)}^{2}=\sum_{j+|\alpha|=k}\left\|\Lambda^{j} \psi_{\alpha}\right\|_{L^{2}\left(S_{\infty}\right)}^{2} .
$$

Finally, we let $W A_{k}^{2}\left(S_{\infty}\right)$ be the Hilbert space obtained by taking the closure, in this norm, of the arrays generated by smooth functions.

Next, we define a space $W A_{k}^{2,1}\left(S_{\infty}\right)$ which is the closure of the arrays $\left(\left.D^{\alpha} \psi\right|_{S_{\infty}}\right.$ : $|\alpha| \leq k)$ for $\psi \in C_{0}^{\infty}\left(\mathbf{R}^{n} \times(0, \infty)\right)$ in the norm

$$
\|\vec{\psi}\|_{W A_{k}^{2,1}\left(S_{\infty}\right)}^{2}=\sum_{j+|\alpha|=k}\left\|\Lambda^{j} \psi_{\alpha}\right\|_{L^{2,1}\left(S_{\infty}\right)}^{2}
$$

where the norm on $L^{2,1}\left(S_{\infty}\right)$ is given by

$$
\|\psi\|_{L^{2,1}\left(S_{\infty}\right)}^{2}=\int_{0}^{\infty} \int_{\partial \Omega}\left|\nabla_{T} \psi(Q, s)\right|^{2}+|\Lambda \psi(Q, s)|^{2} d Q d s .
$$

Here, $\nabla_{T}$ is the tangential component of the spatial gradient. Since we require $\psi$ to vanish at $t=0$, it is easy to see that we have defined a norm and not a semi-norm. These spaces are straightforward generalizations of the arrays used by Greg Verchota [25] to study solutions of the Dirichlet problem for the polyharmonic equation in a Lipschitz domain. To see why these spaces are used, we recall that the standard formulation of the Dirichlet problem allows us to specify $u$ and the normal derivatives up to order $m-1$. If we take the highest order normal derivative, $\partial^{m-1} u / \partial N^{m-1}$, from $L^{2}\left(S_{\infty}\right)$, then the $j$ th normal derivative should have $m-1-j$ spatial derivatives in $L^{2}\left(S_{\infty}\right)$. However, there is no straightforward way to define second and higher order Sobolev spaces on the non-smooth (Lipschitz) surface $S_{\infty}$. In order to overcome this problem, we use the formulation (1.3) of the initialDirichlet problem and the spaces of Whitney arrays.

For a smooth function $u$, we let $\nabla^{j} u$ be the expression

$$
\nabla^{j} u(X, t)=\left(\sum_{|\alpha|=j} \frac{j !}{\alpha !}\left|D^{\alpha} u(X, t)\right|^{2}\right)^{1 / 2} .
$$

As usual, for a multi-index $\alpha, \alpha$ ! is defined as $\alpha_{1} ! \alpha_{2} ! \ldots \alpha_{n} !$. Our estimates for solutions will be given using a parabolic maximal function. To define this, we let

$$
\Gamma_{\beta}(P, t)=\left\{(Y, s):|Y-P|+|t-s|^{1 / 2}<(1+\beta) \delta(Y), Y \in \Omega, 0<s \leq t\right\}
$$


denote the parabolic approach region with vertex at $(P, t)$. Then for $v$ on $\Omega \times(0, \infty)$, we define the parabolic maximal function

$$
v^{*}(P, t)=\sup _{(Y, s) \in \Gamma_{\beta}(P, t)}|v(Y, s)|, \quad(P, t) \in S_{\infty} .
$$

We will assume that $\beta$ is sufficiently large so that $\left\{\left(P+s e_{n}, t\right): s>0\right\} \subset \Gamma_{\beta}(P, t)$. The constants in our estimates may depend on $\beta$. Since $\Omega$ is a Lipschitz graph domain, it is easy to see that this can be done with $\beta$ depending only on $M$, the Lipschitz constant for $\phi$. Finally, we will define restriction to the boundary in the parabolic sense: $D^{\alpha} u=f_{\alpha}$ on $S_{T}$ means that

$$
\lim _{\substack{(Y, s) \rightarrow(Q, t) \\(Y, s) \in \Gamma_{\beta}(Q, t)}} D^{\alpha} u(Y, s)=f_{\alpha}(Q, t),
$$

almost everywhere with respect to surface measure on $S_{T}$. With these definitions, we can give our main results.

Theorem 1.4. Let $L$ be an elliptic operator of order $2 m$ and $\Omega$ a Lipschitz graph domain. Then for each $\vec{\psi} \in W A_{m-1}^{2}\left(S_{\infty}\right)$, the initial-Dirichlet problem (1.3) has a solution satisfying

$$
\left\|\left(\nabla^{m-1} u\right)^{*}\right\|_{L^{2}\left(S_{\infty}\right)} \leq C\|\vec{\psi}\|_{W A_{m-1}^{2}\left(S_{\infty}\right)},
$$

and the functions $D^{\alpha} u,|\alpha| \leq m-1$, have non-tangential limits a.e. on $S_{\infty}$. Furthermore, if $\left(\nabla^{m-1} u\right)^{*} \in L^{2}\left(\Omega_{T}\right)$ for some $T>0$ and $D^{\alpha} u=0$ on $S_{T}$ for $|\alpha| \leq m-1$, then $u=0$ in $\Omega_{T}$.

If the data lies in $W A_{m-1}^{2,1}\left(S_{\infty}\right)$, then the solution satisfies

$$
\left\|\left(\nabla^{m} u\right)^{*}\right\|_{L^{2}\left(S_{\infty}\right)} \leq C\|f\|_{W A_{m-1}^{2,1}\left(S_{\infty}\right)},
$$

and the functions $D^{\alpha} u,|\alpha| \leq m$, have parabolic limits a.e. on $S_{\infty}$. Furthermore, if $\left\|\left(\nabla^{m} u\right)^{*}\right\|_{L^{2}\left(S_{T}\right)}<\infty$, for some $T>0$, and $D^{\alpha} u=0$, on $S_{T}$ for $|\alpha| \leq m-1$, then $u=0$ in $\Omega_{T}$.

The constants in each of these estimates depend only on the Lipschitz constant $M$, the constant $\beta$ appearing in the definition of the approach region, $E, m, n$ and an upper bound for the coefficients.

Both parts of this theorem give estimates for what is usually called the initialDirichlet problem. In recent work, the first problem (given $\vec{\psi} \in W A_{m-1}^{2}\left(S_{\infty}\right)$, find $u$ with $\left.\left(\nabla^{m-1} u\right)^{*} \in L^{2}\left(S_{\infty}\right)\right)$ has come to be called the Dirichlet problem. The second problem (given $\vec{\psi} \in W A_{m-1}^{2,1}\left(S_{\infty}\right)$, find $u$ with $\left(\nabla^{m} u\right)^{*} \in L^{2}\left(S_{\infty}\right)$ ) is called the regularity problem. We will use this terminology below.

There is a long history of boundary value problems on Lipschitz domains. The fundamental papers were due to B. Dahlberg [7] and D. Jerison and C. Kenig 13], who established the $L^{2}$-estimates for the Dirichlet, regularity and Neumann problems for Laplace's equation. Boundary value problems for higher-order elliptic equations were studied first by Dahlberg, Kenig and Verchota [5], who considered the Dirichlet problem for the biharmonic equation. This was extended to higher powers of the Laplacian by G. Verchota [25]. An important breakthrough was achieved by Pipher and Verchota [22], whose boundary Gårding inequality allowed them to attack $2 m$ th order operators directly rather than using the induction scheme developed by Verchota to study powers of the Laplacian. Verchota [26] showed that these methods also could be used to handle elliptic systems. A theory 
for higher order parabolic equations was begun by the first author in collaboration with Shen [3]. Earlier work on second order parabolic equations is due to Fabes and Salsa 9] and the first author [2. The parabolic system associated to Maxwell's equation has been studied in [18]. D. Mitrea, M. Mitrea and J. Pipher have also studied scattering theory for Maxwell's equations [17, 19. This involves equations with complex lower order terms such as we study below.

Following the work of Brown and Shen, most of our effort will be spent studying the elliptic Dirichlet problem

$$
\begin{cases}L u+i \tau u=0, & \text { in } \Omega, \\ D^{\alpha} u(Q)=f_{\alpha}(Q), & |\alpha| \leq m-1, Q \in \partial \Omega,\end{cases}
$$

where $\tau \in \mathbf{R}$ is a parameter. Of course, this boundary value problem arises by applying the Fourier transform in the time variable. By rescaling (and taking the complex conjugate if $\tau<0$ ) we may assume that $\tau=1$. Thus we are led to proving estimates for the Dirichlet problem for the operator $L+i$. While this appears to be a trivial modification of the results of Pipher and Verchota, who studied the Dirichlet problem for the operator $L$, the additional term $i$ introduces new difficulties which cannot be treated by Pipher and Verchota's techniques. In particular, since we will need to rescale to obtain all real values of $\tau$, we cannot view the term involving $i$ as just a lower order perturbation. Our interest in scale-invariant estimates also motivates considering graph domains. These domains provide a simple class of domains which is invariant under rescaling.

The work reported here is an extension of Wei Hu's thesis [12. In particular, the main estimates of this paper and the treatment of the regularity problem are taken directly from [12]. The extension to the Dirichlet problem is new. The outline of this paper is as follows. Sections 2-5 study the boundary value problem for the elliptic equation $L+i \tau$. The last section, section 6 , gives the additional arguments needed to obtain results for the parabolic problem.

Finally, throughout this paper, we assume that constants may depend on the operator through the ellipticity constant $E$, bounds for the coefficients and the order $m$, and that constants may depend on the Lipschitz constant for the function whose graph defines the domain. Also, constants may depend on the parameter $\beta$ defining the approach region. All other dependencies will be noted explicitly.

\section{Estimates for SOlutions of $(L+i) u=0$}

Throughout this section, we assume that $\Omega$ is a Lipschitz graph domain. The operator $L$ is an operator with constant coefficients satisfying the symmetry condition (1.1) and the ellipticity condition (1.2).

We suppose that $u$ is a smooth solution to $(L+i) u=0$ and there is a number $\epsilon>0$ so that, for each multi-index $\alpha$, there is a constant $C_{\alpha}$ so that

$$
\left|D^{\alpha} u(X)\right| \leq C_{\alpha} e^{-\epsilon|X|} .
$$

In Lemma 2.5 we will show that for certain smooth graph domains, the solution of the Dirichlet problem exists and satisfies these estimates. Our main goal here is to derive a priori estimates. 
An important tool in studying solutions of the equation $(L+i) u=0$ is the fundamental solution

$$
\Gamma(X)=\left[\frac{1}{i+\sum a_{\alpha \beta} \xi^{\alpha} \xi^{\beta}}\right]^{\vee},
$$

where the inverse Fourier transform must be understood in the sense of tempered distributions. We note the following estimates for $\Gamma$, which may be proven using standard arguments (or see the second author's thesis [12]). If $2 m-n-|\alpha|<0$, then for each $N \geq 0$,

$$
\left|D^{\alpha} \Gamma(X)\right| \leq \frac{C_{\alpha, N}}{|X|^{|\alpha|+n-2 m}(1+|X|)^{N}}
$$

If $2 m-n-|\alpha|=0$, then for each $N \geq 0$,

$$
\left|D^{\alpha} \Gamma(X)\right| \leq \frac{C_{\alpha, N}}{(1+|X|)^{N}} \log \left(2+\frac{1}{|X|}\right) .
$$

If $2 m-n-|\alpha|>0$, then for each $N \geq 0$,

$$
\left|D^{\alpha} \Gamma(X)\right| \leq \frac{C}{(1+|X|)^{N}}
$$

We now sketch a simple argument to show that we can solve the Dirichlet problem in smooth domains. If this lemma is of interest, it is because we are working in unbounded domains. We say $\Omega$ is a smooth graph domain if $\Omega=\left\{X_{n}>\phi\left(X^{\prime}\right)\right\}$ and $\left\|D^{\alpha} \phi\right\|_{\infty} \leq C_{\alpha},|\alpha| \geq 1$. One can easily see that if we regularize a Lipschitz function $\phi$, the regularizations define a smooth graph domain.

Lemma 2.5. Let $\Omega$ be a smooth graph domain. Then for each $\psi \in C_{0}^{\infty}\left(\mathbf{R}^{n}\right)$, there exists a unique $u \in H^{m}(\Omega)$ with

$$
\left\{\begin{array}{l}
u-\psi \in H_{0}^{m}(\Omega), \\
L u+i u=0
\end{array}\right.
$$

Furthermore, there exists $\epsilon>0$ so that for each multi-index $\alpha$, there exists $C=$ $C(\alpha, \psi)$ for which we have

$$
\left|D^{\alpha} u(X)\right| \leq C e^{-\epsilon|X|}, \quad X \in \Omega .
$$

Proof. We first claim that on any bounded domain $\Omega$, there exists $\epsilon>0$ so that the solution to $(2.6)$ satisfies

$$
\int_{\Omega} e^{\epsilon|X|}\left(|u(X)|^{2}+\left|\nabla^{m} u(X)\right|^{2}\right) d X<C(\psi) .
$$

To see this, observe that for each $j=1, \ldots, n$, choice of + or - , and $\epsilon$ small, the operator

$$
\left(L_{ \pm \epsilon}+i\right)=e^{ \pm \epsilon X_{j}}(L+i) e^{\mp \epsilon X_{j}}
$$

induces a coercive bilinear form on $H_{0}^{m}(\Omega)$. Thus we can apply the Lax-Milgram theorem to conclude that $e^{ \pm \epsilon X_{j}}(u(X)-\psi(X)) \in H_{0}^{m}(\Omega)$, and we have the estimate

$$
\begin{gathered}
\int_{\Omega} e^{ \pm 2 \epsilon X_{j}}\left(|u(X)-\psi(X)|^{2}+\left|\nabla^{m}(u(X)-\psi(X))\right|^{2}\right) d X \\
\leq C \int_{\Omega}\left|\left(L_{ \pm \epsilon}+i\right) \psi(X)\right|^{2} d X .
\end{gathered}
$$


Note that the constant in this estimate depends only on the ellipticity constant and not on the domain. The estimate (2.8) follows easily from (2.9). Now we can apply (2.8) on a sequence of bounded domains $\Omega_{R}=\left\{X_{n}>\phi\left(X^{\prime}\right)\right\} \cap B_{R}(0)$ and conclude that the estimate (2.8) holds on a graph domain. (This does not require $\partial \Omega$ to be smooth.)

Finally, the pointwise estimates (2.7) follow from the $L^{2}$-estimates and standard elliptic regularity theory. This, of course, uses the smoothness of the boundary.

Our first result is taken from Pipher and Verchota, and provides an estimate for the non-tangential maximal function of a solution in terms of its Cauchy data on the boundary.

Proposition 2.10. Suppose $\Omega$ is a Lipschitz graph domain and that $u$ is a solution of $(L+i) u=0$ in $\Omega$, satisfying (2.1). Then we have the estimate

$$
\sum_{j=0}^{2 m-1}\left\|\left(\nabla^{j} u\right)^{*}\right\|_{L^{2}(\partial \Omega)} \leq C \sum_{j=0}^{2 m-1}\left\|\nabla^{j} u\right\|_{L^{2}(\partial \Omega)},
$$

where $C$ depends on $E, M$ and an upper bound for the coefficients.

For the proof below and throughout this paper, we adopt the convention that $D^{j} u D^{k} w$ denotes a sum of the form $\sum_{|\alpha|=j,|\beta|=k} C_{\alpha, \beta}(X) D^{\alpha} u D^{\beta} w$. We use this to keep track of the order of differentiation when the exact form of the expression is unimportant. We continue to use $D^{\alpha}$ for spatial derivatives, where $\alpha$ is a multiindex, and $D_{n}^{k}$ for derivatives with respect to $X_{n}$. In section 6 , when we return to the study of the parabolic problem, $D_{t}^{k}$ will denote derivatives with respect to time.

Proof. We give the estimate for $\left(\nabla^{2 m-1} u\right)^{*}$ and leave it to the reader to adapt the argument to the easier task of estimating the lower order derivatives. We let $\gamma$ and $\delta$ be multi-indices of length $|\gamma|=m-1$ and $|\delta|=m$. We begin with the expression

$$
I=\int_{\Omega} a_{\alpha \beta} D^{\alpha} D^{\gamma} \Gamma(X-Y) D^{\beta} D^{\delta} u(Y) d Y .
$$

In this expression, we first integrate by parts to interchange $D^{\beta}$ and $D^{\gamma}$. If we alternately move one of the differentiations in $D^{\beta}$ to the first term and then take one of the differentiations in $D^{\gamma}$ to the second term, we always obtain a boundary term involving $D^{2 m-1} \Gamma$ and $D^{2 m-1} u$. Thus we conclude that

$$
\begin{aligned}
I=(-1)^{m-1} \int_{\Omega} L \Gamma(X-Y) D^{\gamma+\delta} u(Y) d Y \\
\quad+\int_{\partial \Omega} D^{2 m-1} \Gamma(X-Q) D^{2 m-1} u(Q) d \sigma(Q),
\end{aligned}
$$

where of course $L \Gamma$ must be interpreted as a distribution. In a similar way, we can interchange the $D^{\alpha}$ and $D^{\delta}$ derivatives and obtain

$$
\begin{aligned}
I=(-1)^{m} & \int_{\Omega} D^{\delta} D^{\gamma} \Gamma(X-Y) L u(Y) d Y \\
& +\int_{\partial \Omega} D^{2 m-1} \Gamma(X-Q) D^{2 m-1} u(Q) d Q .
\end{aligned}
$$


Now we equate these expressions, use the facts that $L u+i u=0$ and $L \Gamma+i \Gamma=\delta_{X}$, the delta measure, to obtain

$$
\begin{aligned}
D^{\gamma+\delta} u(X)-i \int_{\Omega} u(Y) D^{\gamma+\delta} & \Gamma(X-Y)-(-1)^{2 m-1} \Gamma(X-Y) D^{\gamma+\delta} u(Y) d Y \\
& =\int_{\partial \Omega} D^{2 m-1} \Gamma(X-Q) D^{2 m-1} u(Q) d Q .
\end{aligned}
$$

If we integrate the derivative $D^{\gamma+\delta}$ by parts we obtain that the remaining integral in $\Omega$ is equal to a boundary potential,

$$
\begin{gathered}
\int_{\Omega} u(Y) D^{\gamma+\delta} \Gamma(X-Y)-(-1)^{2 m-1} \Gamma(X-Y) D^{\gamma+\delta} u(Y) d Y \\
=\int_{\partial \Omega} \sum_{j=0}^{2 m-2} D^{j} u(Q) D^{2 m-2-j} \Gamma(X-Q) d Q .
\end{gathered}
$$

Thus, from (2.11) and (2.12) we obtain the representation formula

$$
\begin{aligned}
D^{\gamma+\delta} u(X)= & \int_{\partial \Omega} \sum_{j=0}^{2 m-2} D^{j} u(Q) D^{2 m-2-j} \Gamma(X-Q) \\
& +D^{2 m-1} \Gamma(X-Q) D^{2 m-1} u(Q) d Q .
\end{aligned}
$$

We have represented $u$ using two types of potentials on the boundary. The first is

$$
T_{2 m-1} f(X)=\int_{\partial \Omega} D^{2 m-1} \Gamma(X-Q) f(Q) d Q,
$$

and by the $L^{2}$-boundedness of the Cauchy integral on Lipschitz curves [4], one obtains

$$
\left\|\left(T_{2 m-1} f\right)^{*}\right\|_{L^{2}(\partial \Omega)} \leq\|f\|_{L^{2}(\partial \Omega)} .
$$

The second type of potential is

$$
T_{j} f(X)=\int_{\partial \Omega} D^{j} \Gamma(X-Q) f(Q) d Q, \quad 0 \leq j \leq 2 m-2 .
$$

By the estimates for the fundamental solution (2.2), (2.3) and (2.4), it is easy to show that the potential in (2.15) satisfies

$$
\left(T_{j} f\right)^{*}(P) \leq C M(f)(P)
$$

where $M$ is the Hardy-Littlewood maximal function. Hence, we have $L^{p}$-estimates

$$
\left\|\left(T_{j} f\right)^{*}\right\|_{L^{p}(\partial \Omega} \leq C_{p}\|f\|_{L^{p}(\partial \Omega)}, \quad 1<p \leq \infty, j=0, \ldots, 2 m-2 .
$$

The estimate of our proposition follows if we use the estimates (2.14) and (2.16) for the potential operators to estimate the non-tangential maximal function of the right-hand side of the representation formula (2.13).

\section{Rellich inequalities} by

In this section, we consider a solution of $(L+i) u=0$ and define integrals of $u$

$$
u_{-k}\left(X^{\prime}, X_{n}\right)=-\int_{X_{n}}^{\infty} u_{1-k}\left(X^{\prime}, t\right) d t \quad \text { and } \quad u_{0}=u .
$$

Note that if $u$ is a solution satisfying (2.1), then $u_{-k}$ also satisfies (2.1). 
Our goal is to show that if $u$ is a solution to the Dirichlet problem with data $\vec{\psi}$, then we have

$$
\begin{aligned}
\sum_{j=0}^{2 m-1}\left\|\nabla^{j} u_{-m}\right\|_{L^{2}(\partial \Omega)} & \leq C\|\vec{\psi}\|_{W A_{m-1}^{2}(\partial \Omega)}, \\
\sum_{j=0}^{2 m-1}\left\|\nabla^{j} u_{1-m}\right\|_{L^{2}(\partial \Omega)} & \leq C\|\vec{\psi}\|_{W A_{m-1}^{2,1}(\partial \Omega)} .
\end{aligned}
$$

Our route to these estimates is not direct. Following Pipher and Verchota, we prove such estimates using an integration by parts argument which is a natural extension of the Rellich identity. These arguments involve the quadratic form for the operator $a_{\alpha \beta} D^{\alpha} u D^{\beta} \bar{u}$ integrated over the boundary. As is well-known, for higher order elliptic operators, we do not necessarily have the pointwise bound

$$
a_{\alpha \beta} D^{\alpha} u(X) D^{\beta} \bar{u}(X) \geq C\left|\nabla^{m} u(X)\right|^{2}
$$

(this always holds if $m=1$ ). Thus it is nontrivial to obtain estimates for $\nabla^{m} u$ in terms of $a_{\alpha \beta} D^{\alpha} u D^{\beta} \bar{u}$. The first estimate we will use is the boundary Gårding inequality of Pipher and Verchota 22. Note that this inequality is just a statement about smooth functions on $\partial \Omega$. In the theorem below, $u$ does not need to solve a partial differential equation. In the next theorem and below, $N_{n}$ denotes the last component of the unit outer normal to the boundary $\partial \Omega$. A key feature of Lipschitz graph domains is that there exists a positive number $\delta$ so that $0<\delta<-N_{n}(Q) \leq 1$ a.e.

Theorem 3.3 (Pipher and Verchota). Let $u$ be a smooth function satisfying (2.1). Then we have

$$
\int_{\partial \Omega}\left|\nabla^{m} u(Q)\right|^{2} d Q \leq C \int_{\partial \Omega} a_{\alpha \beta} D^{\alpha} u(Q) D^{\beta} \bar{u}(Q)\left(-N_{n}(Q)\right)+\left|\nabla^{m-1} D_{n} u(Q)\right|^{2} d Q .
$$

This estimate, and a suitable induction scheme, allow us to exchange general derivatives for $D_{n}$ derivatives on the right-hand side of (3.1) and (3.2), provided we can obtain estimates for $\int_{\partial \Omega} a_{\alpha \beta} D^{\alpha} u D^{\beta} u$. We give a lemma which provides the first step in estimating the quadratic form. After this lemma, we make a few remarks indicating why the operator $L+i$ is substantially different from the operator $L$ that was treated by Pipher and Verchota.

In this lemma and below, we use $D_{T} D^{j} u$ for expressions which are sums of terms of the form $a(Q) \cdot \nabla D^{\alpha} u$, where the vector field $a(Q)$ satisfies $a(Q) \cdot N(Q)=0$ and $\alpha$ is a multi-index of length $j$.

Lemma 3.4. Let $(L+i) u=0$ in a Lipschitz graph domain and assume that $u$ satisfies (2.1). If $\gamma$ is a multi-index of length $m-1$, then we have

$$
\begin{gathered}
\int_{\partial \Omega} a_{\alpha \beta} D^{\alpha} D^{\gamma} u(Q) D^{\beta} D^{\gamma} \bar{u}(Q)\left(-N_{n}(Q)\right) d Q \\
=\int_{\partial \Omega} D^{m-1} D^{\gamma} D_{n} u(Q) D^{2 m-1} \bar{u}(Q) d Q \\
\quad+2(-1)^{m} \operatorname{Re} \int_{\Omega} D^{2 \gamma} D_{n} u(X) i \bar{u}(X) d X
\end{gathered}
$$


and

$$
\begin{gathered}
\int_{\partial \Omega} a_{\alpha \beta} D^{\alpha} D^{\gamma} u(Q) D^{\beta} D^{\gamma} \bar{u}(Q)\left(-N_{n}(Q)\right) d Q \\
=\int_{\partial \Omega} D^{2 m-1} u(Q) D_{T} D^{m-1} D^{\gamma} \bar{u}(Q) d Q \\
\quad+2(-1)^{m} \operatorname{Re} \int_{\Omega} i u(X) D^{2 \gamma} D_{n} \bar{u}(X) d X .
\end{gathered}
$$

Proof. To prove both identities, we begin with the form on the boundary and apply the divergence theorem to obtain

$$
\begin{aligned}
\int_{\partial \Omega} a_{\alpha \beta} D^{\alpha} D^{\gamma} u(Q) & D^{\beta} D^{\gamma} \bar{u}(Q)\left(-N_{n}(Q)\right) d Q \\
& =-2 \operatorname{Re} \int_{\Omega} a_{\alpha \beta} D^{\alpha} D^{\gamma} D_{n} u(X) D^{\beta} D^{\gamma} \bar{u}(X) d X .
\end{aligned}
$$

We may integrate by parts in two different ways in this integral to obtain the two identities (3.5) and (3.6). First, we establish (3.5).

If we integrate by parts to exchange the $D^{\alpha}$ derivative on $u$ and the $D^{\gamma}$ derivative on $\bar{u}$ (see [22, §3] or the proof of Proposition 2.10), we obtain

$$
\begin{aligned}
\int_{\Omega} a_{\alpha \beta} D^{\alpha} D^{\gamma} D_{n} u(X) D^{\beta} D^{\gamma} \bar{u}(X) d X & \\
= & (-1)^{m-1} \int_{\Omega} D^{2 \gamma} D_{n} u(X) L \bar{u}(X) d X \\
& \quad+\int_{\partial \Omega} D^{m-1} D^{\gamma} D_{n} u(Q) D^{2 m-1} \bar{u}(Q) d Q .
\end{aligned}
$$

We substitute $L \bar{u}=i \bar{u}$ in (3.8), and then (3.5) follows from (3.7) and (3.8).

To establish (3.6), we return to the integral over $\Omega$ in (3.7). This time, we integrate by parts to exchange the $D^{\beta}$ derivative on $\bar{u}$ with the $D^{\gamma} D_{n}$ derivative on $u$. This gives

$$
\begin{aligned}
\int_{\Omega} a_{\alpha \beta} D^{\alpha} D^{\gamma} D_{n} u(X) D^{\beta} D^{\gamma} \bar{u}(X) d X & \\
= & \quad(-1)^{m} \int_{\Omega} L u(X) D^{2 \gamma} D_{n} \bar{u}(X) d X \\
& \quad+\int_{\partial \Omega} D^{2 m-1} u(Q) D_{t} D^{m-1} D^{\gamma} \bar{u}(Q) d Q .
\end{aligned}
$$

Note that when we interchange derivatives of the same order, we obtain a tangential derivative in the boundary term (see [22, §3]). When the order of differentiation is one, this is clear since

$$
\int_{\Omega} D_{i} F D_{j} G-D_{j} F D_{i} G d X=\int_{\partial \Omega} F\left(N_{i} D_{j} G-N_{j} D_{i} G\right) d Q
$$

and it is easy to see that $N_{i} D_{j}-N_{j} D_{i}$ is a tangential derivative. When the order of differentiation is greater than one, we can use induction to see that we still obtain one tangential derivative. Thus (3.6) follows if we use the equation $L u=-i u$ in (3.9) and substitute the result into (3.7). 
The main new contribution of this paper is to provide estimates for the integral over $\Omega$ in the identities (3.5) and (3.6). We remark that if $u$ satisfies $(L+1) u=0$, then the above argument gives

$$
\begin{aligned}
\int_{\partial \Omega} & \left(a_{\alpha \beta} D^{\alpha} D^{\gamma} u(Q) D^{\beta} D^{\gamma} \bar{u}(Q)+\left|D^{\gamma} u(Q)\right|^{2}\right)\left(-N_{n}(Q)\right) d Q \\
= & \int_{\partial \Omega} D^{2 m-1} u(Q) D^{m-1} D^{\gamma} D_{n} \bar{u}(Q)+\sum_{j=0}^{m-2} D^{j} u(Q) D^{m-2-j} D^{\gamma} D_{n} \bar{u}(Q) d Q .
\end{aligned}
$$

This leads to a proof of (3.1) and (3.2) for solutions of the equation $(L+1) u=0$. This is not the case for the non-self-adjoint operator $L+i$. It is interesting to compare this with the work of Verchota and Vogel [24], who studied general second order equations including non-self-adjoint equations. They have also found that the study of non-self-adjoint equations is more interesting than the self-adjoint case because there is no Rellich identity. Instead, we must prove estimates.

Before continuing with the hard work of estimating the integral over $\Omega$ in (3.5) and (B.6), we give some elementary estimates for solutions of $(L+i) u=0$. We begin with a mean-value inequality. The key feature here is that the constant in this inequality decays rapidly with respect to the radius $R$. We will use $f_{E} f$ to denote the average $|E|^{-1} \int_{E} f$.

Lemma 3.10. Suppose $(L+i) u=0$ in a ball $B_{R}\left(X_{0}\right)$. Then for each multi-index $\alpha$ and $N>0$, there exists a constant $C_{\alpha, N}$ so that

$$
\left|D^{\alpha} u\left(X_{0}\right)\right| \leq \frac{C_{\alpha, N}}{R^{|\alpha|}(1+R)^{N}} f_{B_{R}\left(X_{0}\right)}|u(Y)| d Y .
$$

Proof. We first give the proof in the case $2 m-n<0$, and then we have the estimate (2.2) for the fundamental solution $\Gamma(X)$. We pick a cutoff function $\eta(X)$ with $\eta=1$ on $B_{R / 2}\left(X_{0}\right), \eta$ is supported in $B_{R}\left(X_{0}\right)$ and $\eta$ satisfies the natural estimates $\left|D^{\alpha} \eta\right| \leq C_{\alpha} R^{-|\alpha|}$. Using the fundamental solution to represent $\eta u$ and then differentiating with respect to $X$ gives

$$
D^{\alpha} u(X)=\int_{B_{R}(X)} D^{\alpha} \Gamma(X-Y)(L+i)(\eta u)(Y) d Y, \quad X \in B_{R / 2}\left(X_{0}\right) .
$$

Since $(L+i) u=0$ and $D^{\alpha} \eta=0$ in $B_{R / 2}\left(X_{0}\right)$, we may integrate by parts to move all derivatives to $\eta$ and $\Gamma$, and obtain

$$
D^{\alpha} u(X)=\int_{B_{R}\left(X_{0}\right) \backslash B_{R / 2}\left(X_{0}\right)} \sum_{j=1}^{2 m} D^{j} \eta(Y) D^{2 m-j+|\alpha|} \Gamma(X-Y) u(Y) d Y .
$$

The estimate (3.11) follows using estimates (2.2) for derivatives of $\Gamma$.

The case $2 m-n \geq 0$ may be deduced from the case already considered by adding artificial variables $Z$ and viewing $u$ as a solution of $\left(L+\Delta_{Z}^{2 m}+i\right) u=0$. We omit the details.

Using the mean value inequality 3.11, we obtain two estimates relating the $L^{2}$-norm of $u$ and its derivatives on $\Omega$.

Lemma 3.12. Suppose $u$ is a solution of $(L+i) u=0$ in a Lipschitz graph domain $\Omega$. If $\lim _{X_{n} \rightarrow \infty} u\left(X^{\prime}, X_{n}\right)=0$ for each $X^{\prime} \in \mathbf{R}^{n-1}$, then

$$
\int_{\Omega}|u(X)|^{2} d X \leq C \int_{\Omega}\left|D_{n} u(X)\right|^{2} d X .
$$


Proof. Proof It is convenient to simplify the geometry by mapping $\Omega$ to a half space $\left\{X_{n}>0\right\}$. We let

$$
v\left(X^{\prime}, t\right)=u\left(X^{\prime}, \phi\left(X^{\prime}\right)+t\right)
$$

and observe that $D_{t} v\left(X^{\prime}, t\right)=D_{n}\left(X^{\prime}, \phi\left(X^{\prime}\right)+t\right)$ and $D_{t} v$ still satisfies the meanvalue property

$$
\left|D_{t} v\left(X^{\prime}, t\right)\right| \leq \frac{C}{(1+t)^{N}}\left(f_{B_{t / 2}\left(X^{\prime}, t\right)}\left|D_{s} v(Y, s)\right|^{2} d Y^{\prime} d s\right)^{1 / 2}
$$

for all $N \geq 0$. We write

$$
v\left(X^{\prime}, t\right)=-\int_{t}^{\infty} D_{s} v\left(X^{\prime}, s\right) d s,
$$

and apply Hardy's inequality (see Stein [23, p. 272) and the mean-value inequality, to obtain

$$
\int_{0}^{\infty}\left|v\left(X^{\prime}, t\right)\right|^{2} d t \leq C \int_{0}^{\infty}(1+s)^{-2 N} s^{2} f_{B_{s / 2}\left(X^{\prime}, s\right)}\left|D_{r} v\left(Y^{\prime}, r\right)\right|^{2} d Y^{\prime} d r d s .
$$

Now we integrate with respect to $X^{\prime}$ over $\mathbf{R}^{n-1}$ and apply Fubini's theorem to the right-hand side, and obtain

$$
\int_{\mathbf{R}^{n-1}} \int_{0}^{\infty}\left|v\left(X^{\prime}, t\right)\right|^{2} d X^{\prime} d t \leq C \int_{0}^{\infty} \int_{\mathbf{R}^{n-1}}\left|D_{r} v\left(Y^{\prime}, r\right)\right|^{2} r^{2}(1+r)^{-2 N} d Y^{\prime} d r .
$$

Thus we can choose $N=1$, and the lemma follows.

Our next lemma gives the opposite estimate, almost.

Lemma 3.13. Suppose $u$ satisfies $(L+i) u=0$. Then for each $\epsilon>0$ and each multi-index $\alpha$, there exists a constant $C=C_{\epsilon, \alpha}$ so that

$$
\int_{\Omega \cap\{\delta(X)>\epsilon\}}\left|D^{\alpha} u(X)\right|^{2} d X \leq C_{\epsilon, \alpha} \int_{\Omega}|u(X)|^{2} d X
$$

Proof. The proof depends on the additional decay in the mean value property of Lemma 3.10. Again, we make the change of variables to a half-space and set

$$
v_{\alpha}\left(X^{\prime}, t\right)=\left(D^{\alpha} u\right)\left(X^{\prime}, \psi\left(X^{\prime}\right)+t\right)
$$

and $v\left(X^{\prime}, t\right)=u\left(X^{\prime}, \psi\left(X^{\prime}\right)+t\right)$. Then Lemma 3.10 implies that

$$
\left|v_{\alpha}\left(X^{\prime}, t\right)\right| \leq t^{-|\alpha|}\left(f_{B_{t / 2}\left(X^{\prime}, t\right)}\left|v\left(Y^{\prime}, s\right)\right|^{2} d Y^{\prime} d s\right)^{1 / 2}
$$

Squaring this estimate, integrating over $\mathbf{R}^{n-1} \times(\epsilon, \infty)$ and using Fubini's theorem gives

$$
\begin{aligned}
\int_{\epsilon}^{\infty} \int_{\mathbf{R}^{n-1}}\left|v_{\alpha}\left(X^{\prime}, t\right)\right|^{2} d X^{\prime} d t & \leq C_{\alpha} \int_{\epsilon / 2}^{\infty} \int_{\mathbf{R}^{n-1}} s^{-2|\alpha|}\left|v\left(Y^{\prime}, s\right)\right|^{2} d Y^{\prime} d s \\
& \leq C_{\alpha} \epsilon^{-2|\alpha|} \int_{\epsilon / 2}^{\infty} \int_{\mathbf{R}^{n-1}}\left|v\left(Y^{\prime}, s\right)\right|^{2} d Y^{\prime} d s
\end{aligned}
$$


When we apply the above lemma, we will need to estimate functions in a thin strip along the boundary. This is easily accomplished using the non-tangential maximal function. For the elliptic problem, the non-tangential maximal function is defined for functions $v$ on $\Omega$ by

$$
v^{*}(P)=\sup _{X \in \Gamma_{\beta}(P)}|v(X)| .
$$

The approach region is defined by $\Gamma_{\beta}(P)=\{X \in \Omega:|X-P|<(1+\beta) \delta(X)\}$. The parameter $\beta$ is assumed to be large, as in the parabolic case.

Lemma 3.14. Let $u$ be a solution of $(L+i) u=0$ which satisfies (2.1). For each $\epsilon>0$, there exists a constant $C_{\epsilon}$ so that $u$ satisfies the estimate

$$
\begin{aligned}
& \int_{\partial \Omega} \sum_{j=0}^{2 m-2}\left|\nabla^{j} u(Q)\right|^{2} d Q+\int_{\Omega} \sum_{j=0}^{2 m-1}\left|\nabla^{j} u(X)\right|^{2} d X \\
& \quad \leq \int_{\partial \Omega} \epsilon\left(\nabla^{2 m-1} u\right)^{*}(Q)^{2}+C_{\epsilon} \sum_{j=0}^{m-1}\left|\nabla^{j} D_{n}^{m-1} u(Q)\right|^{2} d Q .
\end{aligned}
$$

Proof. Recall that in a graph domain, the last component of the normal, $N_{n}$, is bounded away from zero. We begin the proof by estimating the boundary integral on the left-hand side of the estimate of the lemma. The divergence theorem, Cauchy-Schwarz and Lemma 3.12 give

$$
\begin{aligned}
\int_{\partial \Omega} \sum_{j=0}^{2 m-2}\left|\nabla^{j} u(Q)\right|^{2} d Q & \leq C \int_{\partial \Omega} \sum_{j=0}^{2 m-2}\left|\nabla^{j} u(Q)\right|^{2}\left(-N_{n}(Q)\right) d Q \\
& =2 C \int_{\Omega}^{2 m-2} \sum_{j=0}^{j} \nabla^{j} u(X) D_{n} \nabla^{j} u(X) d X \\
& \leq C \int_{\Omega}\left|\nabla^{2 m-1} u(X)\right|^{2} d X .
\end{aligned}
$$

To estimate the integral over $\Omega$ on the left-hand side of the estimate of the lemma, apply Lemma 3.12 to obtain

$$
\int_{\Omega} \sum_{j=0}^{2 m-1}\left|\nabla^{j} u(X)\right|^{2} d X \leq C \int_{\Omega}\left|\nabla^{2 m-1} u(X)\right|^{2} d X .
$$

Now Lemma 3.13 and Lemma 3.12 give

$$
\begin{aligned}
\int_{\Omega}\left|\nabla^{2 m-1} u(X)\right|^{2} d X \\
\quad \leq \epsilon \int_{\partial \Omega}\left(\nabla^{2 m-1} u\right)^{*}(Q)^{2} d Q+C_{\epsilon} \int_{\Omega}|u(X)|^{2} d X \\
\quad \leq \epsilon \int_{\partial \Omega}\left(\nabla^{2 m-1} u\right)^{*}(Q)^{2} d Q+C_{\epsilon} \int_{\Omega}\left|D_{n}^{m-1} u(X)\right|^{2} d X .
\end{aligned}
$$

We turn our attention to the last term in the above estimate, (3.17). We observe that

$$
\int_{\Omega}\left|D_{n}^{m-1} u(X)\right|^{2} d X \leq\left.\left|\int_{\Omega} i\right| D_{n}^{m-1} u(X)\right|^{2}+a_{\alpha \beta} D^{\alpha} D_{n}^{m-1} u(X) D^{\beta} D_{n}^{m-1} \bar{u}(X) d X \mid .
$$


This holds since the second term inside the absolute value sign is real. Integrating by parts gives

$$
\begin{aligned}
& \int_{\Omega} i\left|D_{n}^{m-1} u(X)\right|^{2}+a_{\alpha \beta} D^{\alpha} D_{n}^{m-1} u(X) D^{\beta} D_{n}^{m-1} \bar{u}(X) d X \\
& =(-1)^{m-1} \int_{\Omega} D_{n}^{2 m-2} \bar{u}(X)(i u(X)+L u(X)) d X \\
& \quad+\int_{\partial \Omega} \sum_{j=0}^{m-2} D_{n}^{m-1+j} u(Q) D^{m-2-j} u(Q)+D^{m-1} D_{n}^{m-1} u(Q) D^{2 m-1} u(Q) d Q .
\end{aligned}
$$

Since $(L+i) u=0$, we have the estimate

$$
\begin{aligned}
& \int_{\Omega}\left|D_{n}^{m-1} u(X)\right|^{2} d X \\
& \leq C\left(\int_{\partial \Omega} \sum_{j=0}^{m-1}\left|\nabla^{j} D_{n}^{m-1} u(Q)\right|^{2} d Q\right)^{1 / 2}\left(\int_{\partial \Omega} \sum_{j=0}^{2 m-1}\left|\nabla^{j} u(Q)\right|^{2} d Q\right)^{1 / 2} .
\end{aligned}
$$

Now using (3.15), (3.17) and (3.18) we obtain

$$
\begin{aligned}
& \int_{\partial \Omega} \sum_{j=0}^{2 m-2}\left|\nabla^{j} u(Q)\right|^{2} d Q \\
& \leq \int_{\partial \Omega} C \epsilon\left(\nabla^{2 m-1} u\right)^{*}(Q)^{2}+C_{\epsilon} / \epsilon \sum_{j=0}^{m-1}\left|\nabla^{j} D_{n}^{m-1} u(Q)\right|^{2} d Q+\epsilon \sum_{j=0}^{2 m-1}\left|\nabla^{j} u(Q)\right|^{2} d Q,
\end{aligned}
$$

where we have applied Young's inequality to obtain the last two terms. In the last term, we move the summands $\nabla^{j} u, j=0, \ldots, 2 m-2$, to the left. The last term, with $j=2 m-1$, we bound above by the non-tangential maximal function. This gives the estimate for the boundary term in the lemma.

To estimate the term in the interior, we use (3.16), (3.17) and (3.18) and then the estimate for the boundary term.

\section{Estimates FOR the REgUlarity PROBLEM}

In this section, we derive the main estimate, (3.2), for the regularity problem. Our goal is to establish the existence of solutions to the regularity problem

$$
\begin{cases}(L+i) u=0, & \text { in } \Omega, \\ D^{\alpha} u=\psi_{\alpha}, & |\alpha| \leq m-1, \\ \left(\nabla^{m} u\right)^{*} \in L^{2}(\partial \Omega) . & \end{cases}
$$

Here, $\vec{\psi}$ lies in the space $W A_{m-1}^{2,1}(\partial \Omega)$ of Whitney arrays defined by taking the closure of the arrays generated by $C_{0}^{\infty}\left(\mathbf{R}^{n}\right)$ functions $\vec{\psi}=\left(\psi_{\alpha}:|\alpha| \leq m-1\right)$ in the norm

$$
\sum_{|\alpha| \leq m-1}\left\|\psi_{\alpha}\right\|_{L^{2,1}(\partial \Omega)}^{2}
$$

We begin with the following lemma. Iterating the estimate of this lemma gives the estimate (3.2). 
Lemma 4.1. Suppose $u$ is a solution of $(L+i) u=0$ in a Lipschitz graph domain and that $u$ satisfies (2.1). Let $\gamma$ be a multi-index with $|\gamma|=m-1$. There exist a constant $C$, and, for each $\epsilon>0$, a constant $C_{\epsilon}$ so that

$$
\begin{aligned}
& \int_{\partial \Omega}\left|\nabla^{m} D^{\gamma} u(Q)\right|^{2} d Q \\
& \leq \int_{\partial \Omega} C\left|\nabla^{m-1} D^{\gamma} D_{n} u(Q)\right|^{2}+C_{\epsilon} \sum_{j=0}^{m-1}\left|\nabla^{j} D_{n}^{m-1} u(Q)\right|^{2}+\epsilon\left(\nabla^{2 m-1} u\right)^{*}(Q)^{2} d Q .
\end{aligned}
$$

Proof. We begin with the boundary Gårding inequality of Pipher and Verchota, Theorem 3.3, which gives

$$
\begin{aligned}
& \int_{\partial \Omega}\left|\nabla^{m} D^{\gamma} u(Q)\right|^{2} d Q \\
& \quad \leq C \int_{\partial \Omega}\left|\nabla^{m-1} D_{n} D^{\gamma} u(Q)\right|^{2}+a_{\alpha \beta} D^{\alpha+\gamma} u(Q) D^{\beta+\gamma} \bar{u}(Q)\left(-N_{n}(Q)\right) d Q .
\end{aligned}
$$

Next, we use the identity (3.5) of Lemma 3.4 to obtain

$$
\begin{aligned}
\left|\int_{\partial \Omega} a_{\alpha \beta} D^{\alpha+\gamma} u(Q) D^{\beta+\gamma} \bar{u}(Q)\left(-N_{n}(Q)\right) d Q\right| \\
\leq \int_{\partial \Omega}\left|D^{2 m-1} u(Q) D^{m-1} D^{\gamma} D_{n} \bar{u}(Q)\right| d Q \\
\quad+\int_{\Omega}\left|D^{2 \gamma} D_{n} u(X) \bar{u}(X)\right| d X .
\end{aligned}
$$

Then, the Cauchy-Schwarz inequality and the estimate of Lemma 3.14 give

$$
\int_{\Omega}\left|u(X) D^{2 \gamma} D_{n} \bar{u}(X)\right| d X \leq \int_{\partial \Omega} C_{\epsilon} \sum_{j=0}^{m-1}\left|\nabla^{j} D_{n}^{m-1} u(Q)\right|^{2}+\epsilon\left(\nabla^{2 m-1} u\right)^{*}(Q)^{2} d Q .
$$

The lemma follows from (4.2), 4.3) and (4.4).

We can now give a preliminary version of (3.2).

Lemma 4.5. Let $u$ be as in Lemma 4.1. Then

$$
\int_{\partial \Omega} \sum_{j=0}^{2 m-1}\left|\nabla^{j} u(Q)\right|^{2} d Q \leq \int_{\partial \Omega} C_{\epsilon} \sum_{j=0}^{m}\left|\nabla^{j} D_{n}^{m-1} u(Q)\right|^{2}+\epsilon\left(\nabla^{2 m-1} u\right)^{*}(Q)^{2} d Q .
$$

Proof. Lemma 3.14, gives the estimate

$$
\int_{\partial \Omega} \sum_{j=0}^{2 m-2}\left|\nabla^{j} u(Q)\right|^{2} d Q \leq \int_{\partial \Omega} C_{\epsilon} \sum_{j=0}^{m-1}\left|\nabla^{j} D_{n}^{m-1}(Q)\right|^{2}+\epsilon\left(\nabla^{2 m-1} u\right)^{*}(Q)^{2} d Q .
$$

Thus we only need to estimate $\int_{\partial \Omega}\left|\nabla^{2 m-1} u(Q)\right|^{2} d Q$. Applying Lemma $4.1 m-1$ times (first to $D^{\gamma} u,|\gamma|=m-1$, then to $D^{\gamma^{\prime}} D_{n} u,\left|\gamma^{\prime}\right|=m-2$, and so on) gives that for every $\epsilon>0$

$$
\int_{\partial \Omega}\left|\nabla^{2 m-1} u(Q)\right|^{2} d Q \leq \int_{\partial \Omega} C_{\epsilon} \sum_{j=0}^{m}\left|\nabla^{j} D_{n}^{m-1} u(Q)\right|^{2}+\epsilon\left(\nabla^{2 m-1} u\right)^{*}(Q)^{2} d Q .
$$

Combining the resulting estimate with (4.6) gives the desired conclusion. 
Our final step is to observe that we may replace $\nabla^{m} D_{n}^{m-1} u$ by $\nabla_{T} \nabla^{m-1} D_{n}^{m-1} u$ on the right-hand side of the inequality in Lemma 4.5 The expression $\nabla_{T} \nabla^{j} u$ is defined as $\left(\sum_{|\alpha|=j}\left|\nabla_{T} D^{\alpha}\right|^{2}\right)^{1 / 2}$. This argument is taken directly from Pipher and Verchota 22]. We repeat the details for the convenience of the reader. Let $v$ be a smooth function defined near $\partial \Omega$ which satisfies (2.1) and let $\gamma$ be a multi-index of length $m$. By the ellipticity assumption (1.2), we have

$$
E\left|D^{\gamma} u(Q)\right|^{2} \leq a_{\alpha \beta} N^{\alpha}(Q) N^{\beta}(Q)\left|D^{\gamma} u(Q)\right|^{2} .
$$

Now we write $\alpha=\alpha^{\prime}+e_{i}$, and $\gamma=\gamma^{\prime}+e_{j}$. Note that $N_{i} D_{j} u-N_{j} D_{i} u$ is a tangential derivative; thus we have

$$
a_{\alpha \beta} N^{\alpha} N^{\beta}\left|D^{\gamma} u\right|^{2} \leq a_{\alpha \beta} N^{\alpha^{\prime}} N^{\beta} N_{j} D_{X_{i}} D^{\gamma^{\prime}} u D^{\gamma} \bar{u}+\left|\nabla_{T} \nabla^{m-1} u\right|\left|\nabla^{m} u\right| .
$$

Repeating this argument a total of $2 m$ times, we obtain

$$
E\left|D^{\gamma} u\right|^{2} \leq a_{\alpha \beta} D^{\alpha} u D^{\beta} \bar{u} N^{2 \gamma}+C\left|\nabla_{T} \nabla^{m-1} u\right|\left|\nabla^{m} u\right| .
$$

Now we multiply by the binomial coefficient, $m ! / \gamma$ !, and sum over all $\gamma,|\gamma|=m$, to obtain

$$
E\left|\nabla^{m} u\right|^{2} \leq a_{\alpha \beta} D^{\alpha} u D^{\beta} \bar{u}|N|^{2 m}+C\left|\nabla_{T} \nabla^{m-1} u\right|\left|\nabla^{m} u\right| .
$$

We multiply this pointwise estimate by $-N_{n}$, integrate over $\partial \Omega$ and then apply Young's inequality with $\epsilon$ 's to obtain

$$
\begin{aligned}
& \int_{\partial \Omega}\left|\nabla^{m} u(Q)\right|^{2}\left(-N_{n}(Q)\right) d Q \\
& \quad \leq C \int_{\partial \Omega}\left|\nabla_{T} \nabla^{m-1} u(Q)\right|^{2}+a_{\alpha \beta} D^{\alpha} u(Q) D^{\beta} \bar{u}(Q)\left(-N_{n}(Q)\right) d Q .
\end{aligned}
$$

We are now ready to give

Proposition 4.9. Suppose $(L+i) u=0$ and that $u$ satisfies (2.1). For each $\epsilon>0$, there is a constant $C_{\epsilon}$ so that

$$
\begin{aligned}
\int_{\partial \Omega} \sum_{j=0}^{2 m-1}\left|\nabla^{j} u(Q)\right|^{2} d Q \leq \int_{\partial \Omega} & C_{\epsilon}\left(\left|\nabla_{T} \nabla^{m-1} D_{n}^{m-1} u(Q)\right|^{2}\right. \\
& \left.+\sum_{j=0}^{m-1}\left|\nabla^{j} D_{n}^{m-1} u(Q)\right|^{2}\right)+\epsilon\left(\nabla^{2 m-1} u\right)^{*}(Q)^{2} d Q
\end{aligned}
$$

Proof. We begin with the conclusion of Lemma 4.5 We need to estimate the term

$$
\int_{\partial \Omega}\left|\nabla^{m} D_{n}^{m-1} u(Q)\right|^{2} d Q
$$

which appears in the conclusion of Lemma 4.5. First, we apply the observation (4.8) with $u$ replaced $D_{n}^{m-1} u$ and then the identity (3.6) of Lemma 3.4 to bound the form for $L$, to give

$$
\begin{gathered}
\int_{\partial \Omega}\left|\nabla^{m} D_{n}^{m-1} u\right|^{2} d Q \leq \int_{\partial \Omega} C_{\epsilon}\left|\nabla_{T} \nabla^{m-1} D_{n}^{m-1} u(Q)\right|^{2}+\epsilon\left(\nabla^{2 m-1} u\right)^{*}(Q)^{2} d Q \\
+\int_{\Omega}\left|u(X) D_{n}^{2 m-1} u(X)\right| d X .
\end{gathered}
$$


The integral over $\Omega$ can be estimated by Young's inequality and Lemma 3.14 to give

$$
\int_{\Omega}\left|u(X) D_{n}^{2 m-1} u(X)\right| d X \leq \int_{\partial \Omega} C_{\epsilon} \sum_{j=0}^{m-1}\left|\nabla^{j} D_{n}^{m-1} u(Q)\right|^{2}+\epsilon\left(\nabla^{2 m-1} u\right)^{*}(Q)^{2} d Q .
$$

Using this estimate in (4.10) and then Lemma 4.5 gives the Proposition.

Combining the estimates of Proposition 4.9 and Proposition 2.10 gives

Theorem 4.11. Suppose $\psi \in C_{0}^{\infty}\left(\mathbf{R}^{n}\right)$, and that $\Omega$ is a smooth graph domain. Then there is a smooth solution to $(L+i) u=0$ which satisfies

$$
\sum_{j=0}^{2 m-1}\left\|\left(\nabla^{j} u_{1-m}\right)^{*}\right\|_{L^{2}(\partial \Omega)} \leq C \sum_{j=0}^{m-1}\left\|\nabla^{j} \psi\right\|_{L^{2}(\partial \Omega)}+C\left\|\nabla_{T} \nabla^{m-1} \psi\right\|_{L^{2}(\partial \Omega)}
$$

and $D^{\alpha} u(Q)=D^{\alpha} \psi(Q),|\alpha| \leq m-1, Q \in \partial \Omega$.

Proof. According to Lemma 2.5 there is a smooth solution $u$ to $(L+i) u=0$ which satisfies (2.1) and $D^{\alpha} u=D^{\alpha} \psi,|\alpha| \leq m-1$. If we set

$$
u_{-1}(X)=-\int_{X_{n}}^{\infty} u\left(X^{\prime}, t\right) d t
$$

and then $u_{-j-1}=\left(u_{-j}\right)_{-1}, j \geq 1$, we see that $u_{-j}$ still satisfies (2.1). Thus, we may apply the estimate of Proposition 4.9 and then Proposition 2.10 to obtain

$$
\begin{gathered}
\int_{\partial \Omega} \sum_{j=0}^{2 m-1}\left|\left(\nabla^{j} u_{1-m}\right)^{*}(Q)\right|^{2} d Q \leq \int_{\partial \Omega} C_{\epsilon}\left|\nabla_{T} \nabla^{m-1} \psi(Q)\right|^{2}+C_{\epsilon} \sum_{j=0}^{m-1}\left|\nabla^{j} \psi(Q)\right|^{2} \\
+\epsilon\left(\nabla^{2 m-1} u\right)^{*}(Q)^{2} d Q .
\end{gathered}
$$

Thus, if we choose $\epsilon$ small, we obtain the estimate of this theorem.

Corollary 4.12. If $\vec{\psi} \in W A_{m-1}^{2,1}(\partial \Omega)$ and $\Omega$ is a Lipschitz graph domain, then there is a solution to $(L+i) u=0, D^{\alpha} u=\psi_{\alpha}$ on $\partial \Omega,|\alpha| \leq m-1$, which satisfies

$$
\sum_{j=0}^{2 m-1}\left\|\left(\nabla^{j} u_{1-m}\right)^{*}\right\|_{L^{2}(\partial \Omega)} \leq C\left(\sum_{j=0}^{m-1}\left\|\nabla^{j} \psi\right\|_{L^{2}(\partial \Omega)}+\left\|\nabla_{T} \nabla^{m-1} \psi\right\|_{L^{2}(\partial \Omega)}\right) .
$$

The existence is proven via a limiting argument. The uniqueness is obtained via energy estimates. Note that the estimate $\left(\nabla^{m} u\right)^{*} \in L^{2}(\partial \Omega)$ implies $|u|$ and $\left|\nabla^{m} u\right|$ are in $L^{2}(\Omega)$ via Lemma 3.10. We refer to Pipher and Verchota 22] or section 6 of this paper for similar arguments. The uniqueness is done in some detail for the parabolic problem in section 6 (or see [12]).

\section{The $L^{2}$-Dirichlet PRoblem}

Our next goal is to establish the estimate (3.1), which is needed to solve the Dirichlet problem. Our argument has two steps. The first step is to consider solutions of $(L+i) u=0$ which satisfy $D^{\alpha} u=0,|\alpha| \leq m-2$. For such solutions, we may use an argument of Nečas 20] to obtain the bound

$$
\int_{\Omega}|u(X)|^{2} d X \leq \int_{\partial \Omega}\left|\nabla^{m-1} u(Q)\right|^{2} d Q
$$


Nečas's arguments for fourth order equations are also used in [3]. As we saw in section 3, such estimates are essential to extending Pipher and Verchota's argument to the equation $(L+i) u=0$. The second step is to show that it suffices to consider solutions with special data. This is carried out using our solution of the regularity problem.

The estimate for $u$ on $\Omega$ is obtained by duality. Thus we consider solutions of the problem

$$
\left\{\begin{array}{l}
(L+i) u=f \\
u \in H_{0}^{m}(\Omega)
\end{array}\right.
$$

We say $u$ is a weak solution of this problem if $u \in H_{0}^{m}(\Omega)$ and

$$
\int_{\Omega} a_{\alpha \beta} D^{\alpha} u D^{\beta} \psi+i u \psi d X=\int_{\Omega} f \psi d X
$$

for each $\psi \in C_{0}^{\infty}\left(\mathbf{R}^{n}\right)$.

It is classical that such weak solutions exist; this was used in Lemma 2.5. Our interest here is the following estimate at the boundary.

Proposition 5.1. Suppose that $\Omega$ is a smooth graph domain, that $f \in C_{0}^{\infty}(\Omega)$, and that $u \in H_{0}^{m}(\Omega)$ is a weak solution of $(L+i) u=f$. Then $u$ satisfies

$$
\int_{\partial \Omega}\left|\nabla^{m} u(Q)\right|^{2} d Q \leq C \int_{\Omega}|f(X)|^{2} d X
$$

Proof. We may use the argument from Lemma 2.5 to see that $u$ and all its derivatives decay exponentially as $|X| \rightarrow \infty$ and that $u$ is smooth up to the boundary.

We use (4.7), which states that

$$
E\left|\nabla^{m} u(Q)\right|^{2} \leq C\left|\nabla^{m} u(Q)\right|\left|\nabla_{T} \nabla^{m-1} u(Q)\right|+a_{\alpha \beta} D^{\alpha} u(Q) D^{\beta} \bar{u}(Q) .
$$

But since $u \in H_{0}^{m}(\Omega), \nabla_{T} \nabla^{m-1} u=0$ on $\partial \Omega$. We multiply the estimate (5.2) by $-N_{n}$, integrate over $\partial \Omega$ and apply the divergence theorem to obtain

$$
\begin{aligned}
E \int_{\partial \Omega}\left|\nabla^{m} u(Q)\right|^{2}\left(-N_{n}(Q)\right) d Q & \leq \int_{\partial \Omega} a_{\alpha \beta} D^{\alpha} u(Q) D^{\beta} \bar{u}(Q)\left(-N_{n}(Q)\right) d Q \\
& =-2 \operatorname{Re}\left(\int_{\Omega} a_{\alpha \beta} D^{\alpha} D_{n} u(X) D^{\beta} \bar{u}(X) d X\right) .
\end{aligned}
$$

In the integral over $\Omega$, we may integrate by parts to interchange $D^{\beta}$ and $D_{n}$. When we do this all boundary terms vanish, since $u \in H_{0}^{m}(\Omega)$ and $D^{\alpha} u$ satisfies the estimates (2.1). Thus we obtain

$$
\int_{\Omega} a_{\alpha \beta} D^{\alpha} D_{n} u(X) D^{\beta} \bar{u}(X) d X=\int_{\Omega}(i u(X)-f(X)) D_{n} \bar{u}(X) d X .
$$

Combining the last two inequalities, we obtain

$$
\int_{\partial \Omega}\left|\nabla^{m} u(Q)\right|^{2} d Q \leq C \int_{\Omega}|u(X)|^{2}+|\nabla u(X)|^{2}+|f(X)|^{2} d X .
$$

Finally, we may use the energy estimate for $u$ and interpolation inequalities to bound

$$
\int_{\Omega}|u(X)|^{2}+|\nabla u(X)|^{2} d X \leq C \int_{\Omega}|f(X)|^{2} d X
$$

This implies the proposition. 
Next, we obtain a dual estimate for solutions of $(L+i) u=0$.

Lemma 5.3. Let $\Omega$ be a smooth graph domain and suppose $u$ satisfies $(L+i) u=0$, $\left(\nabla^{m-1} u\right)^{*} \in L^{2}(\Omega)$ and $D^{\alpha} u=0$ a.e. on $\partial \Omega,|\alpha| \leq m-2$. Then

$$
\int_{\Omega}|u(X)|^{2} d X \leq C \int_{\partial \Omega}\left|\nabla^{m-1} u(Q)\right|^{2} d Q .
$$

Proof. We choose $f \in C_{0}^{\infty}\left(\mathbf{R}^{n}\right)$ and let $v$ be the solution of $(L+i) v=f, v \in H_{0}^{m}(\Omega)$, from Proposition 5.1. We let $u_{h}(X)=u\left(X+h e_{n}\right), h>0$. If we write $f=(L+i) v$ in the integral below and then integrate by parts, we obtain

$$
\begin{aligned}
\int_{\Omega} u_{h}(X) f(X) d X \\
\quad=\int_{\Omega} v(X)(L+i) u_{h}(X) d X+\sum_{j=0}^{2 m-1} \int_{\partial \Omega} D^{j} u_{h}(Q) D^{2 m-1-j} v(Q) d Q .
\end{aligned}
$$

We have $D^{2 m-1-j} v=0$, for $j=m, \ldots, 2 m-1$, since $v \in H_{0}^{m}(\Omega)\left(\right.$ and $\left.v \in C^{\infty}(\bar{\Omega})\right)$. Of course, $(L+i) u_{h}=0$, and if we let $h \rightarrow 0^{+}$then $\left\|\nabla^{j} u_{h}\right\|_{L^{2}(\partial \Omega)} \rightarrow 0, j=$ $0, \ldots, m-2$. This follows since we assume that $D^{\alpha} u=0$ for $|\alpha| \leq m-2$ and $\left(\nabla^{m-1} u\right)^{*} \in L^{2}$. Using these observations and then Proposition 5.1, we get

$$
\left|\int_{\Omega} u(X) f(X) d X\right| \leq C\|f\|_{L^{2}(\Omega)}\left\|\nabla^{m-1} u\right\|_{L^{2}(\partial \Omega)} .
$$

Taking the sup over all $f$ in $C_{0}^{\infty}(\Omega)$, say, we obtain the lemma.

We now consider general smooth Dirichlet data. Thus, let $\Omega$ be a smooth graph domain, let $\psi \in C_{0}^{\infty}\left(\mathbf{R}^{n}\right)$, let $\vec{\psi}$ be the array generated by $\psi$ and let $u$ be the solution of the Dirichlet problem with data $\vec{\psi}$ from Lemma 2.5. We claim that we can find an array $\tilde{\psi}$ in $W A_{m-1}^{2,1}(\partial \Omega)$ so that $D^{\alpha} \psi=\tilde{\psi}_{\alpha+e_{n}},|\alpha| \leq m-2$. To see this, let $\left(D^{\alpha} \psi:|\alpha| \leq m-1\right)$ be the array generated by $\psi$. According to Corollary 4.12, we may solve the regularity problem for $\Delta^{m-1}+i$ (here $\Delta$ is the Laplacian):

$$
\begin{cases}\left(\Delta^{m-1}+i\right) v=0, & \text { in } \Omega, \\ D^{\alpha} v=D^{\alpha} \psi, & |\alpha| \leq m-2 .\end{cases}
$$

If we consider $v_{-1}$, then the estimates of Corollary 4.12 for the regularity problem give

$$
\sum_{|\alpha| \leq m-1}\left\|D^{\alpha} v_{-1}\right\|_{L^{2}(\partial \Omega)}^{2} \leq C\|\vec{\psi}\|_{W A_{m-2}^{2,1}(\partial \Omega)} \leq C\|\vec{\psi}\|_{W A_{m-1}^{2}(\partial \Omega)}
$$

Thus we let $\tilde{\psi} \in W A_{m-1}^{2,1}(\Omega)$ be the array $\left(D^{\alpha} v_{-1}:|\alpha| \leq m-1\right)$. Note that $v$ and hence $v_{-1}$ decay exponentially as $|X| \rightarrow \infty$ (see (2.1)). Thus, we can show that the array $\tilde{\psi}$ is the limit in $W A_{m-1}^{2,1}(\partial \Omega)$ of arrays generated by $\eta_{R}(X) v_{-1}\left(X+h e_{n}\right)$ for appropriate pairs $(h, R)$. Here $\eta_{R}(X)=\eta(X / R)$, and $\eta(X) \in C_{0}^{\infty}\left(\mathbf{R}^{n}\right)$ is a function which is one on $B_{1}(0)$ and supported in $B_{2}(0)$. Now let $\tilde{v}$ be the solution of the regularity problem for $L+i$ with data $\tilde{\psi}=\left(\tilde{\psi}_{\alpha}:|\alpha| \leq m-1\right)$. Then we have

$$
D^{\alpha} D_{n} \tilde{v}=D^{\alpha} \psi, \quad|\alpha| \leq m-2,
$$

and thus

$$
w=u-D_{n} \tilde{v}
$$


is a solution of the Dirichlet problem with special Dirichlet data. Furthermore, by the triangle inequality, Corollary 4.12 and (5.4)

$$
\left\|\nabla^{m-1} w\right\|_{L^{2}(\partial \Omega)} \leq C\left(\left\|\nabla^{m-1} u\right\|_{L^{2}(\partial \Omega)}+\left\|\nabla^{m-1} D_{n} \tilde{v}\right\|_{L^{2}(\partial \Omega)}\right) \leq C\|\psi\|_{W A_{m-1}^{2}(\partial \Omega)} .
$$

Now, according to Lemma 5.3 and (5.5),

$$
\int_{\Omega}|w(X)|^{2} d X \leq C \int_{\partial \Omega}\left|\nabla^{m-1} w(Q)\right|^{2} d Q \leq C\|\psi\|_{W A_{m-1}^{2}(\partial \Omega)}
$$

Also, an argument with the mean-value inequality of Lemma 3.10 gives

$$
\begin{aligned}
\int_{\Omega}\left|D_{n} \tilde{v}(X)\right|^{2} d X & \leq C \int_{\partial \Omega}\left(\nabla^{m} \tilde{v}\right)^{*}(Q)^{2} d Q \\
& \leq C\|\tilde{\psi}\|_{W A_{m-1}^{2,1}(\partial \Omega)}^{2} \leq C\|\psi\|_{W A_{m-1}^{2}(\partial \Omega)}^{2}
\end{aligned}
$$

Since $u=D_{n} \tilde{v}+w$, we conclude from (5.7) and (5.6) that

$$
\int_{\Omega}|u(X)|^{2} d X \leq C\|\psi\|_{W A_{m-1}^{2}(\partial \Omega)}^{2} .
$$

We have proven the following.

Theorem 5.8. Let $\Omega$ be a smooth graph domain, let $\psi \in C_{0}^{\infty}\left(\mathbf{R}^{n}\right)$ and let $u$ solve the Dirichlet problem in $\Omega$ with data $\left(D^{\alpha} \psi:|\alpha| \leq m-1\right)$. Then

$$
\int_{\Omega}|u(X)|^{2} d X \leq C\|\psi\|_{W A_{m-1}^{2}(\partial \Omega)}^{2} .
$$

Now we continue with the main arguments in the proof of (3.1).

Theorem 5.9. Suppose $(L+i) u=0$, and for some $\psi \in C_{0}^{\infty}\left(\mathbf{R}^{n}\right)$ suppose that $D^{\alpha} u=D^{\alpha} \psi$, on $\partial \Omega,|\alpha| \leq m-1, \psi \in C_{0}^{\infty}\left(\mathbf{R}^{n}\right)$. Then we have

$$
\sum_{j=0}^{2 m-1} \int_{\partial \Omega}\left|\nabla^{j} u_{-m}(Q)\right|^{2} d Q \leq \sum_{j=0}^{m-1} \int_{\partial \Omega}\left|\nabla^{j} D_{n}^{m} u_{-m}(Q)\right|^{2} d Q
$$

Proof. We begin by repeating the conclusion of Corollary 4.12, which implies that

$$
\sum_{j=0}^{2 m-1} \int_{\partial \Omega}\left|\nabla^{j} u_{-m}(X)\right|^{2} d X \leq C \sum_{j=0}^{m} \int_{\partial \Omega}\left|\nabla^{j} D_{n}^{m-1} u_{-m}(X)\right|^{2} d X .
$$

We need to introduce one additional $D_{n}$ derivative on the right of this inequality. Towards this end, let $v=u_{-m}$ and apply the boundary Garding inequality of Pipher and Verchota (see Theorem 3.3) to $D_{n}^{m-1} v$, to obtain

$$
\begin{aligned}
\int_{\partial \Omega} & \left|\nabla^{m} D_{n}^{m-1} v(Q)\right|^{2} d Q \\
& \leq \int_{\partial \Omega} a_{\alpha \beta} D^{\alpha} D_{n}^{m-1} v(Q) D^{\beta} D_{n}^{m-1} \bar{v}(Q)\left(-N_{n}(Q)\right)+\left|\nabla^{m-1} D_{n}^{m} v(Q)\right|^{2} d Q
\end{aligned}
$$

The second term in the integral is what we want; thus we must estimate the form for $L$ on the boundary. We begin with the identity 3.5 with $D^{\gamma} u$ replaced by 
$D_{n}^{m-1} v$. If we integrate by parts in the integral over $\Omega$, we obtain

$$
\begin{gathered}
\int_{\partial \Omega} a_{\alpha \beta} D^{\alpha} D_{n}^{m-1} v(Q) D^{\beta} D_{n}^{m-1} \bar{v}(Q)\left(-N_{n}(Q)\right) d Q \\
=\int_{\partial \Omega} D^{m-1} D_{n}^{m} v(Q) D^{2 m-1} \bar{v}(Q)+\sum_{j=0}^{m-2} D_{n}^{m+j} v(Q) D_{n}^{m-2-j} \bar{v}(Q) d Q \\
-2 \operatorname{Re} \int_{\Omega} D_{n}^{m} v(X) i D_{n}^{m-1} \bar{v}(X) d X
\end{gathered}
$$

Now, we consider the integral over $\Omega$ in the last term and use Cauchy-Schwarz, Lemma 3.12 and then Theorem 5.8 to obtain

$$
\begin{aligned}
\left|\int_{\Omega} D_{n}^{m-1} v(X) D_{n}^{m} \bar{v}(X) d X\right| & \leq C \int_{\Omega}\left|D_{n}^{m} v(X)\right|^{2} d X \\
& \leq C \sum_{|\alpha| \leq m-1} \int_{\partial \Omega}\left|D^{\alpha} D_{n}^{m} v(Q)\right|^{2} d Q
\end{aligned}
$$

Now the estimates (5.11), (5.12), and (5.13) give

$$
\begin{aligned}
\int_{\partial \Omega}\left|\nabla^{m} D_{n}^{m-1} v(Q)\right|^{2} d Q \leq C \int_{\partial \Omega} & \left|\nabla^{m-1} D_{n}^{m} v(Q)\right|\left|D^{2 m-1} v(Q)\right| \\
& +\sum_{j=0}^{m-2}\left|D^{m+j} v(Q)\right|\left|D^{m-2-j} v(Q)\right| d Q .
\end{aligned}
$$

Now we give the argument needed to bound the terms with $j=0, \ldots, m-1$ on the right-hand side of (5.10). Fix $\gamma$, a multi-index with $|\gamma| \leq m-1$. Using Lemma 3.12, Lemma 3.13 and Theorem [5.8, we obtain that for every $\epsilon>0$ there is a constant $C_{\epsilon}>0$ so that

$$
\begin{aligned}
\int_{\partial \Omega} & \left|D^{\gamma} D_{n}^{m-1} v(Q)\right|^{2}\left(-N_{n}(Q)\right) d Q=-2 \operatorname{Re} \int_{\Omega} D^{\gamma} D_{n}^{m} v(X) D^{\gamma} D_{n}^{m-1} \bar{v}(X) d X \\
& \leq C \int_{\Omega}\left|D^{\gamma} D_{n}^{m} u(X)\right|^{2} d X \\
& \leq \int_{\partial \Omega} \epsilon\left(\nabla^{|\gamma|+m} v\right)^{*}(Q)^{2}+C_{\epsilon} \sum_{j=0}^{m-1} \mid \nabla^{j} D_{n}^{m} v(Q)^{2} d Q .
\end{aligned}
$$

We sum this inequality for $|\gamma| \leq m-1$, and use the result and (5.14) to estimate the right-hand side of (5.10). If we choose $\epsilon$ small, we obtain the theorem. Note that our estimates for solutions with nice data, (2.7), imply that the non-tangential maximal function lies in $L^{2}$.

We are now ready to prove our main result for the Dirichlet problem.

Theorem 5.15. Let $\vec{\psi} \in W A_{m-1}^{2}(\partial \Omega)$. Then there exists a solution to

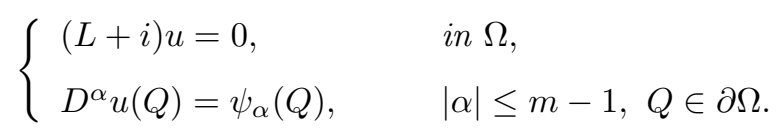

This solution satisfies the estimate

$$
\sum_{j=0}^{2 m-1}\left\|\left(\nabla^{j} u\right)^{*}\right\|_{L^{2}(\partial \Omega)} \leq C\|\vec{\psi}\|_{W A_{m-1}^{2}(\partial \Omega)} .
$$


Furthermore, there is only one solution to the Dirichlet problem (5.16) which also satisfies $\left(\nabla^{m-1} u\right)^{*} \in L^{2}(\partial \Omega)$

The existence follows from the existence of smooth solutions satisfying the estimate (5.17) as established in Theorem 5.9 Note that the solutions from Theorem 5.9 satisfy the estimate (5.17) for the non-tangential maximal function thanks to Proposition 2.10. The uniqueness assertion may be proven by imitating the arguments from Pipher and Verchota 22]; or see the corresponding argument for parabolic equations in $\S 6$. Given the uniqueness result and the estimate (5.17), a limiting argument leads to the existence of solutions for general data. See [22, §9] or section 6 of this paper.

\section{The initial-Dirichlet PROBlem For PARABOLIC EQUATIONS}

Now that we have established our estimates for solutions of the Dirichlet problem for elliptic equations, we turn to the study of parabolic equations. As an intermediate step, we state scaled versions of our estimates for the elliptic problem.

Theorem 6.1. Let $\tau \neq 0, \tau \in \mathbf{R}$.

a) If $\vec{\psi} \in W A_{m-1}^{2}(\partial \Omega)$, then there exists a solution to $(L+i \tau) u=0, D^{\alpha} u=\psi_{\alpha}$, and it satisfies the estimate

$$
\begin{aligned}
& \sum_{j=0}^{2 m-1} \int_{\partial \Omega}|\tau|^{(2 m-1-j) / m}\left(\nabla^{j} u_{-m}\right)^{*}(Q)^{2} d Q \\
& \quad \leq C \int_{\partial \Omega} \sum_{|\alpha| \leq m-1}|\tau|^{(m-1-|\alpha|) / m}\left|\psi_{\alpha}(Q)\right|^{2} d Q .
\end{aligned}
$$

b) If $\vec{\psi} \in W A_{m-1}^{2,1}(\partial \Omega)$, we may solve the regularity problem $(L+i \tau) u=0$, $D^{\alpha} u=\psi_{\alpha},|\alpha| \leq m-1$, and we have the estimate

$$
\begin{aligned}
& \sum_{j=0}^{2 m-1} \int_{\partial \Omega}|\tau|^{(2 m-1-j) / m}\left(\nabla^{j} u_{1-m}\right)^{*}(Q)^{2} d Q \\
& \quad \leq C \sum_{|\alpha| \leq m-1} \int_{\partial \Omega}\left|\psi_{\alpha}(Q)\right|^{2}|\tau|^{(m-|\alpha|) / m} d Q+C \int_{\partial \Omega} \sum_{|\alpha|=m-1}\left|\nabla_{T} \psi_{\alpha}(Q)\right|^{2} d Q .
\end{aligned}
$$

Next, we sketch the details needed to go from the scaled estimates of Theorem 6.1 to the existence of solutions to the initial-Dirichlet problem

$$
\begin{cases}D_{t} u+L u=0, & \text { in } \Omega \times(0, \infty), \\ u(X, 0)=0, & X \in \Omega, \\ D^{\alpha} u(Q, t)=\psi_{\alpha}(Q, t), & |\alpha| \leq m-1,(Q, t) \in \partial \Omega \times(0, \infty) .\end{cases}
$$

Here, $\left(\psi_{\alpha}:|\alpha| \leq m-1\right)$ lies in the space $W A_{m-1}^{2,1}(\partial \Omega \times(0, \infty))$ which was defined in the introduction. To complete the definition, we define the fractional time derivative $\Lambda$ by

$$
\widehat{\Lambda f}(\tau)=(i \tau)^{1 / 2 m} \hat{f}(\tau)
$$


where the root is defined for $\operatorname{Re} z \geq 0$ by $\left(e^{i \theta} r\right)^{1 / 2 m}=r^{1 / 2 m} e^{i \theta / 2 m}$, and $-\pi / 2 \leq$ $\theta \leq \pi / 2$. Note that this operation is given by

$$
\Lambda f(t)=c_{m} D_{t} \int_{0}^{t} f(s)(t-s)^{\frac{-1}{2 m}} d s,
$$

where $c_{m}=\frac{1}{\pi} \sin \left(\frac{\pi}{2 m}\right)$, and we have $\Lambda^{2 m}=D_{t}$.

Our strategy for studying the parabolic boundary value problems is as follows. We begin with a nice solution which is obtained by energy estimates. Since we assume that $u$ has zero initial data, we may extend $u$ to $\Omega \times \mathbf{R}$ by assigning $u$ the value zero for $t<0$. The resulting function is a smooth solution of $D_{t} u+L u=0$ in $\Omega \times \mathbf{R}$. We apply the Fourier transform in the time variable and then use the estimates of Theorem 6.1. Thanks to Plancherel's theorem, this immediately gives estimates for $u$. Using these estimates and a representation formula as in Proposition 2.10, we obtain estimates for the non-tangential maximal function.

We begin by observing that if the array $\vec{\psi}$ is generated by a $C_{0}^{\infty}\left(\mathbf{R}^{n} \times(0, \infty)\right)$ function, then there exists a solution $u$ to (6.2) which satisfies

$$
\int_{0}^{\infty} \int_{\Omega}\left|\nabla^{m} u(X, t)\right|^{2} d X d t+\sup _{t} \int_{\Omega} u(X, t)^{2} d X<\infty
$$

(see Friedman's monograph [10]). Furthermore, if $\Omega$ is smooth, then $u$ extends smoothly up to the boundary.

Next, we observe that the equation $D_{t}+L$ has a fundamental solution $\Gamma(X, t)$ [10] which satisfies

$$
\left|D_{t}^{k} D_{x}^{\alpha} \Gamma(X, t)\right| \leq \frac{C}{t^{n / 2 m+|\alpha| / 2 m+k}} \exp \left(-\frac{1}{C}\left(\frac{|X|^{2 m}}{t}\right)^{\frac{1}{2 m-1}}\right)
$$

for multi-indices $\alpha$ and integers $k$ with $|\alpha| \geq 0$ and $k=0,1,2, \ldots$ In this estimate, $C$ is a constant depending on $\alpha, k, E, m, n$ and an upper bound for the coefficients. Using this fundamental solution, we can prove interior estimates for solutions in a manner similar to Lemma 3.10. This argument shows that if $D_{t} u+L u=0$ in $J_{r}(X, t)=B_{r}(X) \times\left(t-r^{2 m}, t\right]$, then for any multi-index $\alpha$ and $k=0,1,2, \ldots$

$$
\left|D_{t}^{k} D_{x}^{\alpha} u(X, t)\right| \leq C r^{-n-2 m(k+1)-|\alpha|} \iint_{J_{r}(X, t)}|u(Y, s)| d Y d s,
$$

where $C$ does not depend on $r$. These estimates imply that if $u$ is a solution satisfying the energy estimate (6.3), then for each $X \in \Omega$, multi-index $\alpha$, and $k=1,2, \ldots$ we have that $D^{\alpha} D_{t}^{k} u(X, \cdot) \in L^{2}(\mathbf{R})$, and if $|\beta| \geq m$, then $D^{\beta} u(X, \cdot) \in$ $L^{2}(\mathbf{R})$. Thus we may define the partial Fourier transform in the time variable, and for $\tau \neq 0$ we have

$$
\hat{u}(X, \tau)=(i \tau)^{-1} \widehat{D_{t} u}(X, \tau) .
$$

Also, Plancherel's theorem and (6.3) give that for a.e. $\tau$, we have

$$
\int_{\Omega}\left|D^{\alpha} \hat{u}(X, \tau)\right|^{2} d X<\infty, \quad|\alpha|=m
$$

From this estimate and a rescaled version of Lemma 3.12, it follows that

$$
\int_{\Omega}|\hat{u}(X, \tau)|^{2}|\tau| d X \leq C \int_{\Omega}\left|\nabla^{m} \hat{u}(X, \tau)\right|^{2} d X
$$


Thus, $\hat{u}(X, \tau)$ is a weak solution of the Dirichlet problem with data $\hat{\psi}(X, \tau)$. If $\tau \neq 0$, we have proved that weak solutions are unique and hence $\hat{u}$ satisfies the estimates of Theorem 6.1 part a). Applying the inverse Fourier transform gives

$$
\begin{aligned}
& \int_{0}^{\infty} \int_{\partial \Omega} \sum_{|\alpha|+j=2 m-1}\left|\Lambda^{j} D^{\alpha} u_{1-m}(Q, s)\right|^{2} d Q d s \\
& \quad \leq \int_{0}^{\infty} \int_{\partial \Omega} \sum_{|\alpha|+j=m,|\alpha| \leq m-1}\left|\Lambda^{j} D^{\alpha} \psi(Q, s)\right|^{2}+\sum_{|\alpha|=m}\left|\nabla_{T} D^{\alpha} \psi(Q, s)\right|^{2} d Q d s .
\end{aligned}
$$

Similarly, applying the estimates of part b) gives

$$
\begin{aligned}
& \int_{0}^{\infty} \int_{\partial \Omega} \sum_{|\alpha|+j=2 m-1}\left|\Lambda^{j} D^{\alpha} u_{-m}(Q, s)\right|^{2} d Q d s \\
& \quad \leq \int_{0}^{\infty} \int_{\partial \Omega} \sum_{|\alpha|+j=m-1}\left|\Lambda^{j} D^{\alpha} \psi(Q, s)\right|^{2} d Q d s .
\end{aligned}
$$

Next, we observe that we have the following representation formula.

Proposition 6.8. Let $\Omega$ be a smooth domain and let $u(X, t)$ solve

$$
\begin{cases}D_{t} u+L u=0, & \text { in } \Omega \times(0, \infty), \\ u(X, 0)=0, & X \in \Omega, \\ u \in C^{\infty}(\bar{\Omega} \times[0, \infty)), & \end{cases}
$$

and for each $T<\infty$, assume that

$$
\int_{0}^{T} \int_{\Omega}\left|\nabla^{m} u(X, t)\right|^{2} d X d t<\infty
$$

Let $v=u_{-j}$ for $j \geq 0$ and let $|\gamma|=m-1,|\delta|=m$. Then

$$
\begin{array}{r}
D^{\gamma+\delta} v(X, t)=\int_{0}^{t} \int_{\partial \Omega} D^{2 m-1} v(Q, s) D^{2 m-1} \Gamma(X-Q, t-s) \\
+\sum_{j=0}^{2 m-2} D^{j} v(Q, s) D^{2 m-2-j} D_{s} \Gamma(X-Q, t-s) d Q d s .
\end{array}
$$

Proof. We begin by observing that if $u$ satisfies (6.9), then, for each $\epsilon>0, u$ satisfies

$$
|u(X, t)| \leq C(t, u, N, \epsilon) \delta(X)^{-N}, \quad \delta(X)>\epsilon .
$$

To see this, observe that the interior estimates (6.5) and the bound (6.9) imply that for $k \geq 1$,

$$
\begin{aligned}
\left|D_{t}^{k} D^{\alpha} u(X, t)\right| & =\left|D_{t}^{k-1} L D^{\alpha} u(X, t)\right| \\
& \leq C r^{-2 k m-|\alpha|-n / 2}\left(\int_{t-r^{2 m}}^{t} \int_{B_{r}(X)}\left|\nabla^{m} u(Y, s)\right|^{2} d Y d s\right)^{1 / 2} .
\end{aligned}
$$

We choose $r=\delta(X) / 2$ in this bound and conclude that

$$
\left|D_{t}^{k} D^{\alpha} u(X, t)\right| \leq C(u) \delta(X)^{-2 k m-|\alpha|-n / 2} .
$$

Now if we integrate in time, we have

$$
\left|D^{\alpha} u(X, t)\right| \leq C(u) t^{k} \delta(X)^{-2 k m-|\alpha|-n / 2} .
$$


This inequality implies that we can form the integral $u_{-j}$ for all $j$ and that $D^{\alpha} u_{-j}$ decays rapidly in the $X_{n}$-direction, uniformly for $t$ in a finite interval $[0, T]$. In addition, since $\psi$ and $\partial \Omega$ are smooth we have that derivatives of $u_{-j}$ are bounded near the boundary:

$$
\sup _{0<t<T}\left|D_{t}^{k} D^{\alpha} u_{-j}(X, t)\right|<C(\alpha, k, u, j) .
$$

Using these estimates and the estimates for the fundamental solution $\Gamma$ (see 10 . or (6.4)), we may imitate integration by parts arguments in Proposition 2.10 and obtain that for multi-indices $|\gamma|=m-1$ and $|\delta|=m$, we have

$$
\begin{aligned}
& D^{\gamma+\delta} u_{-j}(X, t)=\int_{0}^{t} \int_{\partial \Omega} D^{2 m-1} \Gamma(X-Q, t-s) D^{2 m-1} u_{-j}(Q, s) \\
& +\sum_{k=0}^{2 m-2} D^{k} u_{-j}(Q, s) D^{2 m-2-k} D_{s} \Gamma(X-Q, t-s) d Q d s .
\end{aligned}
$$

Now we quote several results on parabolic maximal functions of the potentials appearing in the representation formula of Proposition 6.8.

Theorem 6.10. Suppose $\Omega$ is a Lipschitz graph domain, $f$ is a function in $L^{p}\left(S_{\infty}\right)$, $1<p<\infty$, and $|\alpha|=2 m-1$, and consider the potential

$$
T_{0} f(X, t)=\int_{0}^{t} \int_{\partial \Omega} f(Q, s) D^{\alpha} \Gamma(X-Q, t-s) d Q d s .
$$

This operator satisfies

$$
\left\|T_{0}(f)^{*}\right\|_{L^{p}\left(S_{\infty}\right)} \leq C\|f\|_{L^{p}\left(S_{\infty}\right)} .
$$

For $k=1, \ldots 2 m-2, f$ satisfying $\Lambda^{k} f \in L^{p}\left(S_{\infty}\right)$ and $\beta$ a multi-index with $|\beta|=$ $2 m-2-k$, consider the potential

$$
T_{k} f(X, t)=\int_{0}^{t} \int_{\partial \Omega} f(Q, s) D_{s} D^{\beta} \Gamma(X-Q, t-s) d Q d s .
$$

This potential satisfies

$$
\left\|T_{k}(f)^{*}\right\|_{L^{p}\left(S_{\infty}\right)} \leq C\left\|\Lambda^{k} f\right\|_{L^{p}\left(S_{\infty}\right)} .
$$

The potentials defined above have parabolic limits almost everywhere on the boundary.

The proof of this theorem for $T_{0}$ depends eventually on the results of Coifman, McIntosh and Meyer [4]. Techniques needed to study parabolic potentials are given in the paper of Fabes and Rivière [8]. The result for the potentials $T_{k}$ is somewhat easier. A proof for the heat equation appears in an appendix to the thesis of Brown [1. A closely related result for the heat equation in non-cylindrical domains, is given by Hofmann and Lewis [11, Lemma 2.14]. In the cylindrical case, the basic $L^{2}$-boundedness result follows easily if we take a partial Fourier transform in time. The estimates in $L^{2}$ and for the non-tangential maximal function are obtained by modifying the standard arguments.

We can now give the existence of solutions with nice data. 
Proposition 6.11. Let $\vec{\psi}$ be an array generated by $\psi \in C_{0}^{\infty}\left(\mathbf{R}^{n} \times(0, \infty)\right)$ and let $\Omega$ be a Lipschitz graph domain. Then there exists a solution of the initial-Dirichlet problem which satisfies the estimate for the regularity problem:

$$
\left\|\left(\nabla^{m} u\right)^{*}\right\|_{L^{2}\left(S_{\infty}\right)}+\sum_{|\alpha|+j=2 m-1}\left\|\Lambda^{j} D^{\alpha} u_{1-m}\right\|_{L^{2}\left(S_{\infty}\right)} \leq C\|\psi\|_{W A_{m-1}^{2,1}\left(S_{\infty}\right)} .
$$

Furthermore, $u$ satisfies the estimate for the $L^{2}$-Dirichlet problem:

$$
\left\|\left(\nabla^{m-1} u\right)^{*}\right\|_{L^{2}\left(S_{\infty}\right)}+\sum_{|\alpha|+j=2 m-1}\left\|\Lambda^{j} D^{\alpha} u_{-m}\right\|_{L^{2}\left(S_{\infty}\right)} \leq C\|\psi\|_{W A_{m-1}^{2}\left(S_{\infty}\right)} .
$$

Proof. On smooth domains, this is an immediate consequence of the estimates (6.6) and (6.7), the representation formula in Proposition 6.8 and the estimates of Theorem 6.10. If $\Omega=\left\{\left(X^{\prime}, X_{n}\right): X_{n}>\phi\left(X^{\prime}\right)\right\}$ is a Lipschitz graph domain, we consider a sequence of smooth domains $\Omega_{k}$. Here, $\Omega_{k}=\left\{\left(X^{\prime}, X_{n}\right): X_{n}>\phi_{k}\left(X^{\prime}\right)\right\}$, and we assume that $\phi_{k} \rightarrow \phi$ uniformly and that $\left\|\nabla \phi_{k}\right\|_{\infty}$ is uniformly bounded. If we let $u_{k}$ be the solution of the initial-Dirichlet problem in $\Omega_{k}$ with data $\vec{\psi}_{k}=$ $\left(\left.D^{\alpha} \psi\right|_{\partial \Omega_{k}}:|\alpha| \leq m-1\right)$, then, according to (6.7), the quantity

$$
\sum_{|\alpha|+j=2 m+1}\left\|\Lambda^{j+1} D^{\alpha}\left(u_{k}\right)_{-m}\right\|_{L^{2}\left(S_{k, \infty}\right)}
$$

is bounded independently of $k$. If we let $\Phi_{k}: \partial \Omega \rightarrow \partial \Omega_{k}$ be the map given by $\Phi_{k}\left(X^{\prime}, \phi\left(X^{\prime}\right)\right)=\left(X^{\prime}, \phi_{k}\left(X^{\prime}\right)\right)$, then we can find a subsequence $u_{k}$ for which we have weak convergence:

$$
\left(D^{\alpha} \Lambda^{j}\left(u_{k}\right)_{-m}\right) \circ \Phi_{k} \rightarrow f_{\alpha, j} .
$$

Using the representation formula of Proposition 6.8 for $u_{k}=D_{n}^{m}\left(u_{k}\right)_{-m}$ and this weak convergence, we have that $u_{k}$ converges locally uniformly to a function $u$ which satisfies the equation $D_{t} u+L u=0$ in $\Omega$ and satisfies the estimates (6.6) and (6.7). The derivatives $D^{\alpha} u,|\alpha|=m$, have parabolic limits a.e. on the boundary. Furthermore, because $\left\|\left(\nabla^{m} u_{k}\right)^{*}\right\|_{L^{2}\left(S_{k, \infty}\right)}$ is bounded independently of $k$, it is easy to see that $D^{\alpha} u=D^{\alpha} \psi,|\alpha| \leq m-1$.

Now a limiting argument, similar to the proof of Proposition 6.11, gives existence of solutions to the regularity problem for general data $\vec{\psi} \in W A_{m-1}^{2,1}\left(S_{\infty}\right)$. Before establishing existence of solutions with data $\vec{\psi} \in W A_{m-1}^{2}\left(S_{\infty}\right)$, we consider the uniqueness statements in Theorem 1.4

Lemma 6.12. If $D_{t} u+L u=0$, then

$$
\left|D^{\alpha} u\left(Q+s e_{n}, t\right)\right| \leq C t s^{-m-|\alpha|} M_{1}\left(\left(\nabla^{m} u\right)^{*}\right)(Q, t) .
$$

In this lemma, $M_{1}$ is the one-dimensional Hardy-Littlewood maximal function in the time variable $t$.

Proof. We write $D_{t} u=-L u$ and use the mean-value inequality (6.5) to write

$$
\begin{aligned}
\left|D^{\alpha} u(X, t)\right| & \leq\left|\int_{0}^{t} D^{\alpha} L u(X, r) d r\right| \\
& \leq C \int_{0}^{t} \delta(X)^{-m-|\alpha|} f f_{J_{c_{0} \delta(X)}(X, r)}\left|\nabla^{m} u(Y, s)\right| d Y d s
\end{aligned}
$$


where $J_{r}(X, r)=B_{r}(X) \times\left(t-r^{2 m}, t\right)$. We choose $c_{0}$ small so that

$$
J_{c_{0} \delta(X)}\left(Q+s e_{n}, r\right) \subset \Gamma(Q, r),
$$

and thus 6.13 gives

$$
\left|D^{\alpha} u\left(Q+s e_{n}, r\right)\right| \leq C s^{-m-|\alpha|} \int_{0}^{t}\left(\nabla^{m} u\right)^{*}(Q, r) d r .
$$

This estimate implies the result of the lemma.

Theorem 6.14. Assume $u$ is a solution of the regularity problem in $\Omega \times(0, T)$ with $D^{\alpha} u=0$ on $\partial \Omega,|\alpha| \leq m-1$, and $\left(\nabla^{m} u\right)^{*} \in L^{2}\left(S_{T}\right)$. Then $u=0$.

Proof. We first claim that $D^{\alpha} u \in L^{2}(\Omega \times(0, T))$ for $|\alpha| \leq m-1$. To see this, we begin with Lemma 6.12 which gives that for any $\alpha$ and $\epsilon>0$, there exists $C_{\epsilon}=C_{\epsilon}(\alpha, T)$ so that

$$
\int_{0}^{T} \int_{\Omega \cap\{\delta(X)>\epsilon\}}\left|D^{\alpha} u(X, t)\right|^{2} d X d t \leq C_{\epsilon} \int_{0}^{T} \int_{\partial \Omega}\left(\nabla^{m} u\right)^{*}(Q, t)^{2} d Q d t .
$$

For $|\alpha| \leq m$, a variant of the Poincaré inequality and (6.15) give

$$
\int_{0}^{T} \int_{\Omega \cap\{\delta(X)<1\}}\left|D^{\alpha} u(X, t)\right|^{2} d X d t \leq C \int_{0}^{T} \int_{\partial \Omega}\left(\nabla^{m} u\right)^{*}(Q, s)^{2} d Q d s .
$$

Recall that the constant in the Poincaré inequality can be chosen to depend on the width of the domain in one direction. Together, these give

$$
\int_{0}^{T} \int_{\Omega}\left|D^{\alpha} u(X, t)\right|^{2} d X d t \leq C \int_{0}^{T} \int_{\partial \Omega}\left(\nabla^{m} u\right)^{*}(Q, t)^{2} d Q d t, \quad|\alpha| \leq m
$$

Next, we observe that the interior estimate (6.5) implies that

$$
\left|D^{\alpha} u\left(Q+s e_{n}, t\right)\right| \leq C_{\alpha} s^{m-|\alpha|}\left(\nabla^{m} u\right)^{*}(Q, t), \quad|\alpha| \geq m .
$$

For $|\alpha| \leq m-1$, we may use the mean-value theorem of calculus and our hypothesis that $D^{\alpha} u$ has parabolic limits zero at a.e. $(Q, t) \in S_{T}$. Together, these imply that

$$
\left|D^{\alpha} u\left(Q+s e_{n}, t\right)\right| \leq C s^{m-|\alpha|}\left(\nabla^{m} u\right)^{*}(Q, s), \quad|\alpha| \leq m .
$$

To establish uniqueness, we consider $u_{\epsilon}(X, t)=u\left(X+\epsilon e_{n}, t\right), \epsilon>0$. Integration by parts and the estimates (6.16) and (6.17) imply

$$
\begin{aligned}
\frac{1}{2} \int_{\Omega} u_{\epsilon}(X, t)^{2} d X & +\int_{0}^{t} \int_{\Omega} a_{\alpha \beta} D^{\alpha} u_{\epsilon}(X, t) D^{\beta} u_{\epsilon}(X, t) d X d t \\
& =\sum_{j=0}^{m-1} \int_{0}^{t} \int_{\partial \Omega} D^{j} u_{\epsilon}(Q, s) D^{2 m-1-j} u_{\epsilon}(Q, s) d Q d s
\end{aligned}
$$

If we let $\epsilon \rightarrow 0^{+}$, then (6.17) and (6.18) imply that the right-hand side of (6.19) goes to zero as $\epsilon \rightarrow 0^{+}$. Thus

$$
0=\frac{1}{2} \int_{\Omega} u(X, t)^{2} d X+\int_{0}^{t} \int_{\Omega} a_{\alpha \beta} D^{\alpha} u(X, t) D^{\beta} u(X, t) d X d t .
$$

Since the inequalities (6.16) and (6.18) imply that $u(\cdot, t) \in H_{0}^{m}(\Omega)$ for a.e. $t$, we have that $\int_{0}^{t} \int_{\Omega} a_{\alpha \beta} D^{\alpha} u D^{\beta} u \geq 0$, and hence (6.20) implies $u=0$. 
Finally, we consider uniqueness in the Dirichlet problem. Here, we use the duality argument as in the elliptic case. Thus we begin with a lemma regarding the existence of solutions to the inhomogeneous initial-Dirichlet problem.

Lemma 6.21. Let $\psi \in C_{0}^{\infty}(\Omega \times(0, T))$. Then there exists a solution to

$$
\begin{cases}D_{t} u+L u=\psi, & \text { in } \Omega \times(0, T), \\ u(X, 0)=0, & \text { in } \Omega, \\ D^{\alpha} u(X, 0)=0, & |\alpha| \leq m-1 .\end{cases}
$$

This solution satisfies $\left(\nabla^{m} u\right)^{*} \in L^{2}\left(S_{T}\right)$.

Proof. We construct $u=v+w$, where $w=\Gamma * \psi$ and $v$ is the solution of the regularity problem with data in the array generated by $-w$. We have $\left(\nabla^{m} w\right)^{*} \in$ $L^{2}\left(S_{T}\right)$, since the fundamental solution and all its derivatives decay rapidly as $|X| \rightarrow \infty$. Now, using estimates for $w$ and its derivatives on $S_{T}$, we can apply Proposition 6.11 to conclude that $\left(\nabla^{m} v\right)^{*} \in L^{2}\left(S_{T}\right)$.

Theorem 6.23. Suppose that $D_{t} u+L u=0,\left(\nabla^{m-1} u\right)^{*} \in L^{2}\left(S_{T}\right)$ and

$$
\begin{cases}D_{t} u+L u=0, & \text { in } \Omega_{T}, \\ u(X, 0)=0, & x \in \Omega, \\ D^{\alpha} u(Q, s)=0, & |\alpha| \leq m-1,(Q, s) \in S_{T}, \\ \left(\nabla^{m-1} u\right)^{*} \in L^{2}\left(S_{T}\right) . & \end{cases}
$$

Then $u=0$.

Proof. Let $f \in C_{0}^{\infty}\left(\Omega_{T}\right)$ and let $v(X, t)$ be the solution of the inhomogeneous problem (6.22) from Lemma 6.21. Then $w(X, t)=v(X, T-t)$ solves the adjoint boundary value problem

$$
\begin{cases}\left(-D_{t}+L\right) w(X, t)=f(X, T-t), & \text { in } \Omega_{T}, \\ D^{\alpha} w(Q, t)=0,|\alpha| \leq m-1, & Q \in \partial \Omega, \\ w(X, T)=0, & X \in \Omega .\end{cases}
$$

Since $\nabla^{j} u=0$ on $S_{T}, j=0, \ldots, m-1$, we may use the mean value theorem of calculus to obtain

$$
\left|\nabla^{j} u\left(Q+h e_{n}, t\right)\right| \leq C h^{m-1-j}\left(\nabla^{m-1} u\right)_{h}^{*}(Q, t),
$$

where $v_{h}^{*}$ denotes the maximal function defined using truncated cones

$$
(v)_{h}^{*}(Q, t)=\sup _{\Gamma_{\beta}(P, t) \cap\{X:|X-P|<h\}} u .
$$

Note that the subscript $h$ denotes a translation for $v$ defined on $\Omega_{T}$, but denotes the truncated maximal function when on $(\nabla v)^{*}$. Also, using interior estimates, we have

$$
\nabla^{m-1+j} u\left(Q+h e_{n}, t\right) \leq C h^{-j}\left(\nabla^{m-1} u\right)_{2 h}^{*}(Q, t)
$$

Since $w$ is a solution near the boundary and $w$ vanishes on $S_{T}$, the same arguments imply that

$$
\nabla^{m+j} w\left(Q+h e_{n}, t\right) \leq C h^{-j}\left(\nabla^{m} w\right)_{h}^{*}(Q, t)
$$


We let $u_{h}(X, t)=u\left(X+h e_{n}, t\right)$; then integrating by parts gives

$$
\begin{aligned}
\int_{0}^{T} & \int_{\Omega} f_{h}(X, T-t) u_{h}(X, t) d X d t \\
& =\int_{0}^{T} \int_{\Omega}\left(-D_{t}+L\right) w_{h}(X, t) u_{h}(X, t) d X d t \\
& =\int_{0}^{T} \int_{\partial \Omega} \sum_{j=0}^{2 m-1} D^{j} w_{h}(Q, t) D^{2 m-1-j} u_{h}(Q, t) d Q d t .
\end{aligned}
$$

Thus from (6.24), 6.25) and (6.26) we have

$$
\begin{aligned}
& \left|\int_{0}^{T} \int_{\partial \Omega} \sum_{j=0}^{2 m-1} D^{j} w_{h}(Q, t) D^{2 m-1-j} u_{h}(Q, t) d Q d t\right| \\
& \quad \leq \int_{0}^{T} \int_{\partial \Omega}\left(\nabla^{m-1} u\right)_{2 h}^{*}(Q, t)\left(\nabla^{m} w\right)^{*}(Q, t) d Q d t .
\end{aligned}
$$

Since $\nabla^{m-1} u=0$ a.e. and $\left(\nabla^{m-1} u\right)^{*}$ is in $L^{2}\left(S_{T}\right)$, it follows from the monotone convergence theorem that $\left(\nabla^{m-1} u\right)_{2 h}^{*} \rightarrow 0$ in $L^{2}\left(S_{T}\right)$. Since also $\left(\nabla^{m} w\right)^{*}$ is in $L^{2}\left(S_{T}\right)$, it is easy to see that the boundary term in (6.27) goes to zero as $h \rightarrow 0^{+}$. Thus we conclude that

$$
\int_{0}^{T} \int_{\Omega} f(X, T-t) u(X, t) d X d t=0 .
$$

Since this holds for every $f$ in $C_{0}^{\infty}\left(\Omega_{T}\right)$, we conclude that $u=0$.

Finally, we are ready to give existence of solutions to the $L^{2}$-Dirichlet problem. Let $\vec{\psi} \in W A_{m-1}^{2}\left(S_{\infty}\right)$ be a smooth function, and suppose that $\vec{\psi}_{j}$ is a sequence of arrays generated by functions in $C_{0}^{\infty}\left(\mathbf{R}^{n} \times(0, \infty)\right)$ and that the sequence converges to $\vec{\psi}$ in $W A_{m-1}^{2}\left(S_{\infty}\right)$. Let $u_{j}$ be the sequence of solutions with data $\vec{\psi}_{j}$ as obtained from Proposition 6.11

According to the uniqueness result (either Theorem 6.14 or 6.23 will do), the difference of two solutions satisfies the estimate

$$
\left\|\left(\nabla^{m-1}\left(u_{j}-u_{k}\right)\right)^{*}\right\|_{L^{2}\left(S_{\infty}\right)} \leq C\left\|\psi_{j}-\psi_{k}\right\|_{W A_{m-1}^{2}\left(S_{\infty}\right)} .
$$

An argument as in the proof of Proposition 6.11 implies that $u_{j}$ converges to a function $u$ which satisfies $\left(\nabla^{m-1} u\right)^{*} \in L^{2}\left(S_{\infty}\right)$, has parabolic limits at the boundary, and thus is a solution to the Dirichlet problem. The estimate (6.28) implies that $u$ has the correct data, $D^{\alpha} u=\psi_{\alpha},|\alpha| \leq m-1$. This completes the proof of the main Theorem [1.4 which was stated in the introduction.

We observe that extensions to $L^{p}$ for $p$ near 2 should be possible as in Pipher and Verchota [22] and Dahlberg and Kenig [6]. One should also be able to obtain results in three dimensions for all $p, 2<p \leq \infty$ (see 22] for elliptic equations and also 21] for the biharmonic equation). The extension to bounded domains is easy and would lengthen this paper without making it more interesting. A more interesting problem is to study these problems in non-cylindrical domains as in Hofmann and Lewis [11] and earlier work of Lewis with J. Silver [14] and M. Murray [15].

Extension of the elliptic results to operators $L+z$ is easy for $z \in \mathbf{C} \backslash\{t+i 0: t<$ $0\}$. The constants depend on the argument of $z$ and blow up as $z$ approaches the 
negative real axis. See C.M. Liu [16] for the second order case, including results on the real axis.

\section{REFERENCES}

[1] Russell M. Brown, Layer potentials and boundary value problems for the heat equation on Lipschitz cylinders, Ph.D. thesis, University of Minnesota, 1987.

[2] - The method of layer potentials for the heat equation in Lipschitz cylinders, Amer. J. Math. 111 (1989), 339-379. MR 90d:35118

[3] Russell M. Brown and Z. Shen, The initial-Dirichlet problem for a fourth-order parabolic equation in Lipschitz cylinders, Indiana Univ. Math. J. 39 (1990), 1313-1353. MR 92b:35075

[4] R.R. Coifman, A. McIntosh, and Y.Meyer, L'intégrale de Cauchy définit un opérateur borné sur $L^{2}$ pour les courbes lipschitziennes, Ann. of Math. 116 (1982), 361-387. MR 82m:42027

[5] B. Dahlberg, C. Kenig, and G. Verchota, The Dirichlet problem for the biharmonic equation in a Lipschitz domain, Annales de I'Institute Fourier Grenoble 36 (1986), fasc. 3, 109-135. MR 88a:35070

[6] B.E.J. Dahlberg and C.E. Kenig, Hardy spaces and the Neumann problem in $L^{p}$ for Laplace's equation in Lipschitz domains, Ann. of Math. 125 (1987), 437-466. MR 88d:35044

[7] Björn E. J. Dahlberg, Estimates of harmonic measure, Arch. Rational Mech. Anal. 65 (1977), no. 3, 275-288. MR 57:6470

[8] E. B. Fabes and N. M. Rivière, Dirichlet and Neumann problems for the heat equation in $C^{1}$-cylinders, Harmonic analysis in Euclidean spaces (Proc. Sympos. Pure Math., Williams Coll., Williamstown, Mass., 1978), Proc. Sympos. Pure Math., XXXV, Part 2, Amer. Math. Soc., Providence, R.I., 1979, pp. 179-196. [MR 81b:35044]

[9] E.B. Fabes and S. Salsa, Estimates of caloric measure and the initial-Dirichlet problem for the heat equation in Lipschitz cylinders, Trans. Amer. Math. Soc. 279 (1983), 635-650. MR 85c:35034

[10] Avner Friedman, Partial differential equations of parabolic type, Prentice-Hall Inc., 1964. MR 31:6062

[11] S. Hofmann and J. Lewis, Solvability and representation by caloric layer potentials in timevarying domains, Ann. of Math. 144 (1996), 349-420. MR 97h:35072

[12] Wei $\mathrm{Hu}$, The initial-boundary value problem for higher order differential operators on Lipschitz cylinders, Ph.D. thesis, University of Kentucky, 1997.

[13] D.S. Jerison and C.E. Kenig, The Neumann problem on Lipschitz domains, Bull. Amer. Math. Soc. 4 (1982), 203-207. MR 84a:35064

[14] J.L. Lewis and J. Silver, Parabolic measure and the Dirichlet problem for the heat equation in two dimensions, Indiana Univ. Math. J. 37 (1988), 801-839. MR 90e:35079

[15] John L. Lewis and Margaret A. M. Murray, The method of layer potentials for the heat equation in time-varying domains, Mem. Amer. Soc. 114 (1995), no. 545. MR 96e:35059

[16] Changmei Liu, The Helmholtz equation on Lipschitz domains, IMA preprint \#1356, 1995.

[17] Dorina Mitrea, Marius Mitrea, and Jill Pipher, Vector potential theory on nonsmooth domains in $\mathbf{R}^{3}$ and applications to electromagnetic scattering, J. Fourier Anal. Appl. 3 (1997), no. 2, 131-192. MR 99e:31009

[18] Marius Mitrea, Initial boundary-value problems for the parabolic Maxwell system in Lipschitz cylinders, Indiana Univ. Math. J. 44 (1995), no. 3, 797-813. MR 96m:35312

[19] _ The method of layer potentials in electromagnetic scattering theory on nonsmooth domains, Duke Math. J. 77 (1995), no. 1, 111-133. MR 96b:78035

[20] Jindrich Nečas, Les methodes directes en theorie des equations elliptique, Masson et Cie., Editeurs, Paris, 1967, Translated from Czech. MR 37:3168

[21] Jill Pipher and Gregory Verchota, The Dirichlet problem in $L^{p}$ for the biharmonic equation on Lipschitz domains, Amer. J. Math. 114 (1992), no. 5, 923-972. MR 94g:35069

[22] Jill Pipher and Gregory C. Verchota, Dilation invariant estimates and the boundary Gårding inequality for higher order elliptic operators, Annals of Math. 142 (1995), 1-38. MR 96g:35052

[23] E.M. Stein, Singular integrals and differentiability properties of functions, Princeton University Press, Princeton NJ, 1970. MR 44:7280

[24] G. C. Verchota and A. L. Vogel, Nonsymmetric systems on nonsmooth planar domains, Trans. Amer. Math. Soc. 349 (1997), no. 11, 4501-4535. MR 98c:35040 
[25] Gregory Verchota, The Dirichlet problem for the polyharmonic equation in Lipschitz domains, Indiana Univ. Math. J. 39 (1990), no. 3, 671-702. MR 91k:35073]

[26] Gregory C. Verchota, Potentials for the Dirichlet problem in Lipschitz domains, Potential theory-ICPT 94 (Kouty, 1994), de Gruyter, Berlin, 1996, pp. 167-187. MR 97f:35041

Department of Mathematics, University of Kentucky, Lexington, Kentucky 40506

E-mail address: rbrown@pop.uky.edu

Department of Mathematics and Computer Science, Houghton College, Houghton, New YoRK 14744

E-mail address: weih@houghton.edu 